\title{
Models of Local Relativistic Quantum Fields with Indefinite Metric (in All Dimensions)
}

\author{
S. Albeverio ${ }^{1,2,3}$, H. Gottschalk ${ }^{1}$, and J.-L. $\mathrm{Wu}^{1,2,4}$ \\ ${ }^{1}$ Fakultät und Institut für Mathematik der Ruhr-Universität Bochum, D-44780 \\ Bochum, Germany \\ 2 SFB237 Essen-Bochum-Düsseldorf, Germany \\ ${ }^{3}$ BiBoS Research Centre, Bielefeld-Bochum, Germany; and CERFIM, Locarno, \\ Switzerland \\ ${ }^{4}$ Probability Laboratory, Institute of Applied Mathematics, Academia Sinica, \\ Beijing 100080, P R China
}

\begin{abstract}
A condition on a set of truncated Wightman functions is formulated and shown to permit the construction of the Hilbert space structure included in the Morchio-Strocchi modified Wightman axioms. The truncated Wightman functions which are obtained by analytic continuation of the (truncated) Schwinger functions of Euclidean scalar random fields and covariant vector (quaternionic) random fields constructed via convoluted generalized white noise, are then shown to satisfy this condition. As a consequence such random fields provide relativistic models for indefinite metric quantum field theory, in dimension 4 (vector case), respectively in all dimensions (scalar case).
\end{abstract}

\section{Introduction}

Since the appearance of gauge theories, it became natural to consider (local) quantum field theory(abbr. QFT) in which not all of the Wightman axioms are satisfied. Such a consideration was in particular natural and also necessary for the study of "charged" fields interacting with gauge fields, because their description conflicts either with locality or with positivity (positive definiteness of the set of Wightman functions [12,18] and [7]). The physical reason for this is that in such theories one must use observables of the charged type which obey a Gauss' law(see e.g. Morchio and Strocchi[13]), instead of using the usual local observables. Actually, from the study of fields such as e.g. $\alpha$-gauge type Higgs models which do not satisfy positivity(see e.g. [11] and references therein), it turned out that it is better in general to keep the locality condition and to give up the positivity condition. This leads to the so called "modified Wightman axioms" of the indefinite metric QFT (see [19]). The difference between indefinite metric QFT and standard (i.e. positive metric) QFT is that 
the axiom of positivity in the latter is replaced by the so-called "Hilbert space structure condition (HSSC)" in the former which permits the construction of Hilbert spaces associated to the given collection of Wightman functions.

In recent years models of Euclidean random fields of scalar and vector type have been constructed via convolution from generalized white noise, see e.g. $[1,2,3,4]$ and references therein. Furthermore, by analytic continuation, one can get Wightman functions from the Schwinger functions of such Euclidean models. The corresponding Wightman functions satisfy the relativistic postulates on invariance, spectral property, locality and cluster property. The positivity condition does not hold, in general, for the Wightman functions, in fact in [2](see also [1]) a counterexample was given to show that the reflection positivity does not hold for the associated Schwinger functions, if the non Gaussian component in the generalized white noise is sufficiently strong. Hence it is very interesting to see whether the Wightman functions of such models satisfy the modified Wightman axioms for indefinite QFT's.

The aim of this paper is to prove that the Wightman functions associated with the above mentioned Euclidean models indeed satisfy the Hilbert space structure condition. Such Euclidean fields provide thus relativistic models for indefinite metric QFT's. The technique required to achieve this is based on explicit formulae for the truncated Wightman functions.

The paper is organized as follows. In Section 1, we introduce majorant Hilbert topologies, a necessary and sufficient condition called Hilbert space structure condition for the existence of a majorant Hilbert topology, and modified Wightman axioms. In Section 2, we present a sufficient Hilbert space structure condition for truncated Wightman functions which implies the Hilbert space structure condition for Wightman functions. In Section 3, we introduce both scalar and vector Euclidean random fields as convoluted generalized white noise. We give explicit formulae for their truncated Wightman functions(we remark that the formulae for the vector models we give in Section 3 are written in a way which is different, although equivalent, from the one used in [3]). We derive them by following the procedure for the scalar models in [2], which makes it possible to prove the temperedness of the truncated Wightman functions(which is a point left open in [3]). Section 4 is devoted to the verification of the Hilbert space structure condition for the models introduced in Section 3.

\section{Majorant Hilbert topologies and modified Wight- man axioms}

In this section, we introduce a majorant Hilbert topology structure associated with Wightman functions. For an extensive mathematical account of such topologies as well as of indefinite inner product spaces, we refer to the monograph Bognár[6]. Here we follow the presentation of [19] and [13]. 
Let $d \in \mathbb{N}$ be a fixed space-time dimension and $q \in \mathbb{N}$ be a fixed number. For any $n \in \mathbb{N}$, let us denote by $\mathcal{S}\left(\mathbb{R}^{d n}, \mathbb{C}^{q^{n}}\right)$ the Schwartz space of all rapidly decreasing $\mathbb{C}^{q^{n}}$-valued $C^{\infty}$-functions on $\mathbb{R}^{d n}$ with the Schwartz topology. Let $\mathcal{S}^{\prime}\left(\mathbb{R}^{d n}, \mathbb{C}^{q^{n}}\right)$ denote its topological dual. Let us begin by introducing the following axioms for Wightman functions $\left\{\mathrm{W}_{n}\right\}_{n \in \mathbb{N}_{0}}$ (with $\mathrm{W}_{0}=1$ for simplicity):

Axiom $\mathbf{I}$ (Temperedness) For any $n \in \mathbb{N}$, the $n$-point function $\mathbf{W}_{n}\left(x_{1}, \cdots, x_{n}\right)$, $x_{1}, \cdots, x_{n} \in \mathbb{R}^{d}$, is a tempered distribution, i.e., $\mathbf{W}_{n} \in \mathcal{S}^{\prime}\left(\mathbb{R}^{d n}, \mathbb{C}^{q^{n}}\right)$.

Axiom II(Poincaré invariance) There is a representation $\mathcal{T}$ of the proper, orthochronous Lorentz group $\mathcal{L}_{+}^{\uparrow}\left(\mathbb{R}^{d}\right)$ (which can be assumed to be irreducible) acting on $\mathbb{R}^{q}$, such that for any $n \in \mathbb{N}$ and any Poincaré transformation $\{a, \Lambda\} \in \mathcal{P}_{+}^{\uparrow}\left(\mathbb{R}^{d}\right)$, the $n$-point function $\mathrm{W}_{n}\left(x_{1}, \cdots, x_{n}\right)$ is invariant under $\{a, \Lambda\}$ :

$$
\mathcal{T}(\Lambda)^{\otimes n} \mathrm{~W}_{n}\left(\Lambda^{-1}\left(x_{1}-a\right), \cdots, \Lambda^{-1}\left(x_{n}-a\right)\right)=\mathrm{W}_{n}\left(x_{1}, \cdots, x_{n}\right),
$$

which should be understood component wise as follows

$$
\begin{aligned}
\mathrm{W}_{n}^{j_{1}, \cdots, j_{n}}\left(x_{1}, \cdots, x_{n}\right) & =\sum_{l_{1}, \cdots, l_{n}=1}^{q} \mathcal{T}(\Lambda)_{l_{1}}^{j_{1}} \cdots \mathcal{T}(\Lambda)_{l_{n}}^{j_{n}} \\
& \times \mathbf{W}_{n}^{l_{1}, \cdots, l_{n}}\left(\Lambda^{-1}\left(x_{1}-a\right), \cdots, \Lambda^{-1}\left(x_{n}-a\right)\right) .
\end{aligned}
$$

We remark that by Axiom II, every $\mathrm{W}_{n}$ is actually a distribution in the difference variables, i.e. there is a tempered distribution $\mathbf{w}_{n} \in \mathcal{S}^{\prime}\left(\mathbb{R}^{d(n-1)}, \mathbb{C}^{q^{n}}\right)$ defined as

$$
\mathrm{w}_{n}\left(y_{1}, \cdots, y_{n-1}\right):=\mathrm{W}_{n}\left(x_{1}, \cdots, x_{n}\right)
$$

where $y_{j}:=x_{j}-x_{j+1}, 1 \leq j \leq n-1$. For $r \in \mathbb{N}$ we adopt the conventions in Vol.II of [16] for the (component wise) definition of the Fourier transform ^ on $\mathcal{S}\left(\mathbb{R}^{d n}, \mathbb{C}^{r}\right)$ and $\mathcal{S}^{\prime}\left(\mathbb{R}^{d n}, \mathbb{C}^{r}\right)$, respectively.

Axiom III(Spectral condition) For any $n \in \mathbb{N}$, the Fourier transform $\hat{\mathbf{w}}_{n}\left(q_{1}, \cdots, q_{n-1}\right)$ is supported in the backward cones

$$
\left\{\left(q_{1}, \cdots, q_{n-1}\right) \in \mathbb{R}^{d(n-1)}: q_{j}^{2} \geq 0, q_{j}^{0}<0,1 \leq j \leq n-1\right\},
$$

where $q_{j}=\left(q_{j}^{0}, \overrightarrow{q_{j}}\right) \in \mathbb{R} \times \mathbb{R}^{d-1}$, and $q_{j}^{2}:=\left|q_{j}^{0}\right|^{2}-\left|\overrightarrow{q_{j}}\right|^{2}$ is in Minkowski metric. (A different sign convention on the Fourier transform in most of the physical literature leads to the interchange of forward and backward cones.)

Axiom IV(Locality) For $n \geq 2$, if $\left(x_{j+1}-x_{j}\right)^{2}<0$ for some $j \in\{1, \cdots, n-$ $1\}$, then

$$
\mathrm{W}_{n}\left(x_{1}, \cdots, x_{j}, x_{j+1}, \cdots, x_{n}\right)= \pm t_{(j, j+1)} \mathrm{W}_{n}\left(x_{1}, \cdots, x_{j+1}, x_{j}, \cdots, x_{n}\right) .
$$


Here + corresponds to integer spin of $\mathcal{T}$, whereas - corresponds to half-integer spin [18]. $t_{(j, j+1)}$ acts on $\mathrm{W}_{n}=\left(\mathrm{W}_{n}^{l_{1}, \ldots, l_{n}}\right)_{l_{1}, \ldots, l_{n}=1, \ldots, q}$ by transposing the indexes $l_{j}$ and $l_{j+1}$.

Let $\underline{\mathcal{S}}$ be the Borchers algebra over $\mathcal{S}\left(\mathbb{R}^{d}, \mathbb{C}^{q}\right)$, namely,

$$
\underline{\mathcal{S}}:=\left\{F=\left(f_{0}, f_{1}, \cdots\right): f_{0} \in \mathbb{C}, f_{n} \in \mathcal{S}\left(\mathbb{R}^{d n}, \mathbb{C}^{q^{n}}\right), n \in \mathbb{N}\right\}
$$

with addition and multiplication given as follows

$$
\begin{gathered}
F+G=\left(f_{0}+g_{0}, f_{1}+g_{1}, \cdots\right) \\
F \otimes G=\left((F \otimes G)_{0},(F \otimes G)_{1}, \cdots\right)
\end{gathered}
$$

where $(F \otimes G)_{n}:=\sum_{j+l=n} f_{j} \otimes g_{l}, n=0,1,2, \cdots$. The topology on $\underline{\mathcal{S}}$ is the direct sum topology induced by the Schwartz topology of $\mathcal{S}\left(\mathbb{R}^{d}, \mathbb{C}^{q}\right)$. Setting

$$
\underline{\mathrm{W}}(F):=\sum_{n=0}^{\infty} \mathrm{W}_{n}\left(f_{n}\right)
$$

where $\mathrm{W}_{0}\left(f_{0}\right)=1 \cdot f_{0}$ is the product of the complex numbers 1 and $f_{0}$, then $\underline{\mathrm{W}}$ is a linear functional on $\underline{\mathcal{S}}$, called Wightman functional. Furthermore, for $\bar{F}=\left(f_{0}, f_{1}, \cdots\right) \in \underline{\mathcal{S}}$, we define its involution by $F^{*}=\left(f_{0}^{*}, f_{1}^{*}, \cdots\right)$, where $f_{n}^{*}\left(x_{1}, \cdots, x_{n}\right):=\overleftarrow{r} \overline{f_{n}\left(x_{n}, \cdots, x_{1}\right)}$, where $\overleftarrow{r}$ acts on $f=\left(f^{l_{1}, \ldots, l_{n}}\right)_{l_{1}, \ldots, l_{n}=1, \ldots, q}$ by reversing the order of the indexes and the bar denotes complex conjugation. Then $\underline{W}$ determines a sesquilinear form on $\underline{\mathcal{S}}$ as follows

$$
<F, G>_{W}:=\underline{\mathrm{W}}\left(F^{*} \otimes G\right), \quad F, G \in \underline{\mathcal{S}} .
$$

Clearly, $\langle\cdot, \cdot\rangle_{W}$ is hermitian if the Wightman functions $\mathrm{W}_{n}, n \in \mathbb{N}$, satisfy the hermiticity condition

$$
\mathrm{W}_{n}\left(x_{1}, \cdots, x_{n}\right)=\overleftarrow{r} \overline{\mathrm{W}_{n}\left(x_{n}, \cdots, x_{1}\right)}, n \in \mathbb{N}
$$

Hereafter we assume this condition for simplicity.

Now set

$$
\mathcal{N}_{W}:=\left\{F \in \underline{\mathcal{S}}:<F, G>_{W}=0, \forall G \in \underline{\mathcal{S}}\right\}
$$

which is the kernel of $\langle\cdot, \cdot\rangle_{W}$, then the quotient space

$$
\mathcal{D}:=\underline{\mathcal{S}} / \mathcal{N}_{W}
$$

is well defined as an indefinite inner product space(cf. [6] for this notion) with respect to the indefinite inner product induced by $\langle\cdot, \cdot\rangle_{W}$ (we denote the induced product by the same notation). In general, $\left.(\mathcal{D},<\cdot, \cdot\rangle_{W}\right)$ can not be a pre-Hilbert space. However, we may specify some Hilbert inner product which dominates $\langle\cdot, \cdot\rangle_{W}$. To this end, we introduce the following notion 
Definition 1.1 By a majorant Hilbert topology $\tau$ of $\langle\cdot, \cdot\rangle_{W}$ on $\mathcal{D}$ we mean a topology determined by a Hilbert inner product $(\cdot, \cdot)$ on $\mathcal{D}$ such that

$$
\left|<F, G>_{W}\right| \leq(F, F)^{\frac{1}{2}}(G, G)^{\frac{1}{2}}, F, G \in \mathcal{D} .
$$

Remark 1.2 An important property of a majorant Hilbert topology $\tau$ is that from (2), we have

$$
F^{(n)} \stackrel{\tau}{\rightarrow} F \Longrightarrow<F^{(m)}, F^{(n)}>_{W} \rightarrow<F, F>_{W} .
$$

Namely, the topology $\tau$ is strong enough for $\tau$-convergence to imply convergence of all the corresponding Wightman functions with respect to the inner product $<\cdot, \cdot>_{W}$.

From Definition 1.1, $(\mathcal{D},(\cdot, \cdot))$ is a pre-Hilbert space. Setting $\mathcal{H}:=\overline{\mathcal{D}}^{(\cdot, \cdot)}$, then $(\mathcal{H},(\cdot, \cdot))$ is a Hilbert space. By the known Riesz theorem, (2) implies that there exists a bounded self-adjoint operator $T$ on $\mathcal{H}$, hereafter called

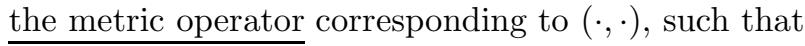

$$
<F, G>_{W}=(F, T G), \quad F, G \in \mathcal{H} .
$$

Moreover, such an operator can be chosen to be non-degenerate, i.e., $T$ fulfills $\mathcal{H}_{T}=\{0\}$, where $\mathcal{H}_{T}:=\{F \in \mathcal{H}:(F, T G)=0, \forall G \in \mathcal{H}\}$ is a Hilbert subspace of $\mathcal{H}$. Actually, suppose that $\mathcal{H}_{T} \neq\{0\}$. We remark that $T\left(\mathcal{H}_{T}\right)=\{0\}$, thus the following Hilbert inner product

$$
(F, G)_{1}:=\left(F,\left(1-\mathbf{P}_{T}\right) G\right)
$$

also determines a majorant Hilbert topology $\tau_{1}$ of $\langle\cdot, \cdot\rangle_{W}$ on $\mathcal{D}$, where $\mathbf{P}_{T}$ : $\mathcal{H} \rightarrow \mathcal{H}_{T}$ is the projection. Clearly, the metric operator $T_{1}:=\left(1-\mathbf{P}_{T}\right)^{-1} T$ is non-degenerate(corresponding to $\left.(\cdot, \cdot)_{1}\right)$. We call such a $\tau_{1}$ a non-degenerate majorant Hilbert topology.

In addition, such a procedure of removing the degeneracy of metric operators also removes the nontrivial ideals of the Borchers algebra $\underline{\mathcal{S}}$ arising from properties like locality and spectral conditions of Wightman functions. On the other hand, we can (well) define a field operator(i.e., an operator valued distribution) $\phi(f)$ on the dense domain $\mathcal{D} \subset \mathcal{H}$ for any $f \in \mathcal{S}\left(\mathbb{R}^{d}, \mathbb{C}^{q}\right)$ as follows

$$
(\phi(f))(G):=F_{f} \otimes G+\mathcal{N}_{W}, G \in \mathcal{D}
$$

where $F_{f}:=(0, f, 0, \cdots) \in \underline{\mathcal{S}}$, with the property that

$$
\begin{aligned}
\mathrm{W}_{n}\left(x_{1}, \cdots, x_{n}\right) & =\left(\Omega, T \phi\left(x_{1}\right) \cdots \phi\left(x_{n}\right) \Omega\right) \\
& =\left(\phi\left(x_{j}\right) \cdots \phi\left(x_{1}\right) \Omega, T \phi\left(x_{j+1}\right) \cdots \phi\left(x_{n}\right) \Omega\right) \\
& =<\phi\left(x_{j}\right) \cdots \phi\left(x_{1}\right) \Omega, \phi\left(x_{j+1}\right) \cdots \phi\left(x_{n}\right) \Omega>_{W}
\end{aligned}
$$


where $\Omega:=(1,0, \cdots)+\mathcal{N}_{W}$. Clearly, $\langle\Omega, \Omega\rangle_{W}>0$.

Since $\mathcal{D}$ by definition of $\phi$ is a multiplication core for the field $\phi$, products of field operators $\phi(f) \phi(g), f, g \in \mathcal{S}\left(\mathbb{R}^{d}, \mathbb{C}^{q}\right)$, are well-defined on $\mathcal{D}$. By Axiom IV and equation (3) the field operators $\phi(f)$ are local in the sense that

$$
[\phi(f), \phi(g)]_{\mp}=0
$$

if the support of the test functions $f, g \in \mathcal{S}\left(\mathbb{R}^{d}, \mathbb{C}^{q}\right)$ is space- like separated. Here $[\cdot, \cdot]_{\mp}$ stands for the commutator if the spin of $\mathcal{T}$ is integer and for the anticommutator otherwise (cf. Axiom IV).

By equation (3) and the hermiticity condition, we conclude that the field

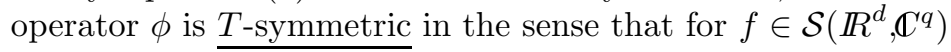

$$
T \phi(\bar{f})^{*} T^{-1}=\phi(f) .
$$

Furthermore, from the action of $\mathcal{P}_{+}^{\uparrow}($ via $\mathcal{T})$ on the test function spaces $\mathcal{S}\left(\mathbb{R}^{d n}, \mathbb{C}^{q^{n}}\right)$ we get a representation $\mathcal{U}$ of the proper orthochronous Poincaré group $\mathcal{P}_{+}^{\uparrow}\left(\mathbb{R}^{d}\right)$ by $T$-unitary operators defined on the common dense domain $\mathcal{D}$, where, by definition, an operator $\mathcal{U}(a, \Lambda)$ on $\mathcal{H}$ is called $T$-unitary, if

$$
\operatorname{TU}(a, \Lambda)^{*} T^{-1}=\mathcal{U}(a, \Lambda)^{-1} .
$$

The field $\phi$ transforms under $\mathcal{U}$ as

$$
\mathcal{U}(a, \Lambda) \phi(x) \mathcal{U}(a, \Lambda)^{-1}=\mathcal{T}(\Lambda) \phi\left(\Lambda^{-1}(x-a)\right) .
$$

Furthermore, the spectral condition in Axiom III is equivalent to the following condition

$$
\int_{\mathbb{R}^{d}}(F, T \mathcal{U}(a, 1) G) e^{i q a} d a=0, F, G \in \mathcal{D}
$$

if $q \notin\left\{q \in \mathbb{R}^{d}: q^{2} \geq 0, q^{0}<0\right\}$.

Now we define a "Krein topology" for $\mathcal{D}$ as follows

Definition 1.3 A non-degenerate majorant Hilbert topology $\tau$ on $\mathcal{D}$ is called a Krein topology if $\mathcal{H}:=\overline{\mathcal{D}}^{\top}$ is maximal. Namely, if $\tau_{1}$ is another non-degenerate

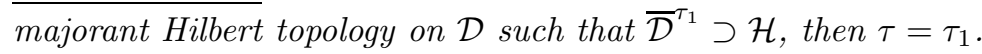

From Definitions 1.1 and 1.3, it is clear that a Krein topology is a minimal topology to provide maximal information from Wightman functions and to keep the density of $\mathcal{D}$ in $\mathcal{H}$. Moreover, we have the following result from [13]:

Proposition 1.4 (Morchio and Strocchi). A majorant Hilbert topology $\tau$ is a Krein topology iff the corresponding metric operator $T$ has a bounded inverse $T^{-1}$. Furthermore, such a bounded invertible operator can be chosen with the property that $T^{2}=1$. 
Given a majorant Hilbert topology $\tau$ with the non-degenerate metric operator $T$ on the Hilbert space $(\mathcal{H},(\cdot, \cdot))$, one can always find a corresponding Krein topology associated with it. To this end, by Proposition 1.4, it is sufficient to find a metric operator with bounded inverse. In fact, remarking that $T$ is selfadjoint and bounded, the absolute operator $|T|$ is well defined. Furthermore,

$$
(F, G)_{K}:=(F,|T| G), \quad F, G \in \mathcal{H}
$$

determines a new Hilbert inner product whose induced Hilbert topology $\tau_{K}$ is weaker than $\tau$ since

$$
(F, F)_{K}=(F,|T| F) \leq\|T\|(F, F), F \in \mathcal{H} .
$$

On the other hand, we have

$$
<F, G>_{W}=(F, T G)=(F,(\operatorname{sign} T) G)_{K}=:\left(F, T_{K} G\right)_{K}, F, G \in \mathcal{H} .
$$

Obviously, $T_{K}^{-1}=T_{K}=\operatorname{sign} T$ is bounded.

Concerning the existence of a majorant Hilbert topology, we have the following crucial condition from [13] and [19]:

Theorem 1.5 (Morchio and Strocchi). Given a collection of Wightman functions $\left\{\mathrm{W}_{n}\right\}_{n \in N_{0}}$, a necessary and sufficient condition for the existence of a majorant Hilbert topology is that the following holds:

Axiom V There is a sequence $\left\{p_{n}\right\}_{n \in \mathbb{N}}$, where $\forall n \in \mathbb{N}, p_{n}: \mathcal{S}\left(\mathbb{R}^{d n}, \mathbb{C}^{q^{n}}\right) \rightarrow$ $[0, \infty)$ is a Hilbert seminorm, such that

$$
\left|\mathrm{W}_{m+n}\left(\varphi^{*} \otimes \eta\right)\right| \leq p_{m}(\varphi) p_{n}(\eta)
$$

3 for all $\varphi \in \mathcal{S}\left(\mathbb{R}^{d m}, \mathbb{C}^{q^{m}}\right), \eta \in \mathcal{S}\left(\mathbb{R}^{d n}, \mathbb{C}^{q^{n}}\right), m, n \in \mathbb{N}$.

Axiom $\mathrm{V}$ is called the Hilbert space structure condition. It is a new axiom for Wightman functions replacing the positivity condition in the standard QFT. The Axioms I-IV together with the Axiom V are called modified Wightman axioms. Such axioms, especially the Hilbert space structure condition, were presented and lucidly discussed in [19].

We remark that, in general, when the Wightman functions do not fulfill the positivity condition, one can not expect in general a unique Hilbert space structure for the states of the theory(the Hilbert space structure depending on the choice of Hilbert seminorms in Axiom V). This is at variance with non indefinite metric QFT, where the positivity condition guarantees the uniqueness of the physical Hilbert space. Uniqueness for indefinite metric QFT can perhaps be restored in terms of scattering theory, see Remark 1.6 below. 
Lastly, let us also present the cluster property for Wightman functions. A sequence of Wightman functions $\left\{\overline{\mathrm{W}}_{n}\right\}_{n \in N_{0}}$ satisfies the cluster property if for any $m, n \in \mathbb{N}$ and any space-like $a \in \mathbb{R}^{d}$ (i.e., $a^{2}<0$ in Minkowski metric)

$$
\begin{aligned}
\mathrm{W}_{m+n}\left(\varphi_{1} \otimes \cdots \otimes \varphi_{m} T_{\lambda a}\left(\varphi_{m+1} \otimes \cdots \otimes \varphi_{m+n}\right)\right) & \stackrel{\lambda \rightarrow \infty}{\longrightarrow} \\
& \mathrm{W}_{m}\left(\varphi_{1} \otimes \cdots \otimes \varphi_{m}\right) \mathrm{W}_{n}\left(\varphi_{m+1} \cdots \otimes \varphi_{m+n}\right)
\end{aligned}
$$

for $\varphi_{1}, \cdots, \varphi_{m+n} \in \mathcal{S}\left(\mathbb{R}^{d}, \mathbb{C}^{q}\right)$, where $T_{\lambda a}$ denotes the representation of the translation by $\lambda a$ on $\mathcal{S}\left(\mathbb{R}^{d n}, \mathbb{C}^{q^{n}}\right)$.

Remark 1.6 We point out that the cluster property of Wightman functions is not an item of the modified Wightman axioms, since it does not (directly) imply the uniqueness of the vacuum and irreducibility of the field algebra as it does in the standard QFT. Nevertheless, the cluster property can still be looked upon as a genuine expression for the physical principle "forces decrease with the (spatial) distance" in indefinite metric QFT. Especially we expect that also in indefinite metric QFT there is a crucial connection between the cluster property and the possibility of an axiomatic scattering theory in such quantum field theories.

\section{A sufficient Hilbert space structure condition for truncated Wightman functions}

Given a sequence of Wightman functions $\left\{\mathrm{W}_{n}\right\}_{n \in \mathbb{N}_{0}}, \mathrm{~W}_{0}=1, \mathrm{~W}_{n} \in \mathcal{S}^{\prime}\left(\mathbb{R}^{d n}, \mathbb{C}^{q^{n}}\right)$, the corresponding sequence of truncated Wightman functions $\left\{\mathrm{W}_{n}^{T}\right\}_{n \in \mathbb{N}}, \mathrm{W}_{n}^{T} \in$ $\mathcal{S}^{\prime}\left(\mathbb{R}^{d n}, \mathbb{C}^{q^{n}}\right)$, is defined recursively by the equations

$$
\mathrm{W}_{n}\left(\varphi_{1} \otimes \cdots \otimes \varphi_{n}\right)=\sum_{I \in \mathcal{P}(n)} \epsilon_{F}(I) \prod_{\left\{j_{1}, \cdots, j_{l}\right\} \in I} \mathrm{~W}_{l}^{T}\left(\varphi_{j_{1}} \otimes \cdots \otimes \varphi_{j_{l}}\right), n \geq 1
$$

where $\varphi_{1}, \cdots, \varphi_{n} \in \mathcal{S}\left(\mathbb{R}^{d}, \mathbb{C}^{q}\right)$ and $\mathcal{P}^{(n)}$ stands for the collection of all partitions $I$ of $\{1, \cdots, n\}$ into disjoint subsets. For each such subset $\left\{j_{1}, \cdots, j_{l}\right\} \in I$ we assume that $j_{1}<\cdots<j_{l} \cdot \epsilon_{F}(I)$ stands for the fermionic parity of the partition $I$, i.e. $\epsilon_{F}(I):=1$ for (bosonic) integer spin $\mathcal{T}$ and

$$
\epsilon_{F}(I):=\prod_{j<l} \operatorname{sign}\left(\pi_{I}(l)-\pi_{I}(j)\right)
$$

for (fermionic) half-integer spin $\mathcal{T}$. For $I=\left\{\left\{j_{1}^{1}, \ldots, j_{l_{1}}^{1}\right\}, \ldots,\left\{j_{1}^{k}, \ldots, j_{l_{k}}^{k}\right\}\right\}$ with $j_{1}^{1}<\ldots<j_{1}^{k}, \pi_{I}$ is defined as the permutation which maps $(1, \ldots, n)$ to $\left(j_{1}^{1}, \ldots, j_{l_{1}}^{1}, \ldots, j_{1}^{k}, \ldots, j_{l_{k}}^{k}\right)$. By the nuclear theorem the sequence of truncated Wightman functions is determined uniquely by the sequence of Wightman functions and vice versa. 
Since the truncated Wightman functions of the models introduced in Section 3 below are much simpler objects than the Wightman distributions themselves, it seems natural to ask for a sufficient condition on the truncated Wightman functions which implies the Hilbert space structure condition(HSSC) for the Wightman functions, as it was introduced in Section 1. The aim of this section is to deduce such a HSSC for truncated Wightman functions which is then verified in Section 4 for both models of Section 3.

Let us first introduce a special system of Schwartz norms $\left\{\|\cdot\|_{K, N}\right\}_{K, N \in \mathbb{N}_{0}}$ on the spaces $\mathcal{S}\left(\mathbb{R}^{d n}, \mathbb{C}^{q^{n}}\right), n \in \mathbb{N}$, by

$$
\|\varphi\|_{K, N}:=\sup _{\substack{x_{1}, \cdots, x_{n} \in \mathbb{R}^{d} \\ 0 \leq\left|\alpha_{1}\right|, \cdots,\left|\alpha_{n}\right| \leq K}}\left|\prod_{l=1}^{n}\left(1+\left|x_{l}\right|^{2}\right)^{N / 2} D^{\alpha_{1} \cdots \alpha_{n}} \varphi\left(x_{1}, \cdots, x_{n}\right)\right|
$$

for $K, N \in \mathbb{N}_{0}$ and $\varphi \in \mathcal{S}\left(\mathbb{R}^{d n}, \mathbb{C}^{q^{n}}\right)$. Here the absolute $|\cdot|$ is induced by the scalar product $<\cdot, \cdot>_{E}^{\otimes n}$ on $\mathbb{C}^{q^{n}} \cong\left(\mathbb{C}^{q}\right)^{\otimes n}$ where $<\cdot, \cdot>_{E}$ stands for the Euclidean scalar product on $\mathbb{C}^{q}, \alpha_{1}, \cdots, \alpha_{n} \in \mathbb{N}_{0}^{d}$ are multi-indexes and for $\alpha_{j}=\left(\alpha_{j}^{0}, \cdots, \alpha_{j}^{d-1}\right)$ we have used the notations $\left|\alpha_{j}\right|=\alpha_{j}^{0}+\cdots+\alpha_{j}^{d-1}$ and

$$
D^{\alpha_{1} \cdots \alpha_{n}}:=D^{\alpha_{1}} \otimes \cdots \otimes D^{\alpha_{n}}
$$

where

$$
D^{\alpha_{j}}:=\frac{\partial^{\left|\alpha_{j}\right|}}{\left(\partial x^{0}\right)^{\alpha_{j}^{0}} \cdots\left(\partial x^{d-1}\right)^{\alpha_{j}^{d-1}}} .
$$

The definition of the Schwartz norms $\|\cdot\|_{K, N}$ clearly implies that for $m, n \in$ $\mathbb{N}_{0}, \varphi \in \mathcal{S}\left(\mathbb{R}^{d m}, \mathbb{C}^{q^{m}}\right), \eta \in \mathcal{S}\left(\mathbb{R}^{d n}, \mathbb{C}^{q^{n}}\right)$ we get

$$
\|\varphi \otimes \eta\|_{K, N}=\|\varphi\|_{K, N}\|\eta\|_{K, N} .
$$

The following lemma shows that the Schwartz norms $\|\cdot\|_{K, N}$ are also well adapted to the operation of taking the tensor product of two tempered distributions:

Lemma 2.1 Let $m, n \in \mathbb{N}, K, N \in \mathbb{N}_{0}$ and $R \in \mathcal{S}^{\prime}\left(\mathbb{R}^{d m}, \mathbb{C}^{q^{m}}\right), S \in \mathcal{S}^{\prime}\left(\mathbb{R}^{d n}, \mathbb{C}^{q^{n}}\right)$. If there exist constants $C_{R}, C_{S}>0$, such that

$$
|R(\varphi)| \leq C_{R}\|\varphi\|_{K, N}, \forall \varphi \in \mathcal{S}\left(\mathbb{R}^{d m}, \mathbb{C}^{q^{m}}\right)
$$

and

$$
|S(\eta)| \leq C_{S}\|\eta\|_{K, N}, \forall \eta \in \mathcal{S}\left(\mathbb{R}^{d n}, \mathbb{C}^{q^{n}}\right)
$$

then

$$
|R \otimes S(\chi)| \leq C_{R} C_{S}\|\chi\|_{K, N}, \forall \chi \in \mathcal{S}\left(\mathbb{R}^{d(m+n)}, \mathbb{C}^{q^{(m+n)}}\right)
$$


Proof By Vol.II(see p.115) of [8] there exist continuous, polynomially bounded functions $F_{R}: \mathbb{R}^{d m} \rightarrow \mathbb{C}^{q^{m}}, F_{S}: \mathbb{R}^{d n} \rightarrow \mathbb{C}^{q^{n}}$ and polynomials $P_{R}, P_{S}$, such that $R=P_{R}(D) F_{R}$ and $S=P_{S}(D) F_{S}$ holds in the sense of tempered distributions. Consequently, for $\chi \in \mathcal{S}\left(\mathbb{R}^{d(m+n)}, \mathbb{C}^{q^{(m+n)}}\right)$, we get that

$$
R \otimes S(\chi)=F_{R} \otimes F_{S}\left(P_{R}(-D) \otimes P_{S}(-D) \chi\right) .
$$

The right hand side(RHS) can be rewritten as an integral over $\mathbb{R}^{d(m+n)}$ where the integrand is a product of a polynomially bounded function with a fast falling function. Thus, the integral converges absolutely and by Fubini's theorem we get

$$
R \otimes S(\chi)=F_{R}\left(P_{R}(-D) \varrho\right)=R(\varrho),
$$

where

$$
\begin{aligned}
\varrho\left(x_{1}, \cdots, x_{m}\right) & :=S\left(\chi\left(x_{1}, \cdots, x_{m}, \cdot\right)\right) \\
& =F_{S}\left(\mathbf{1}_{m} \otimes P_{S}(-D) \chi\left(x_{1}, \cdots, x_{m}, \cdot\right)\right) .
\end{aligned}
$$

Clearly, $\varrho \in \mathcal{S}\left(\mathbb{R}^{d m}, \mathbb{C}^{q^{m}}\right)$. Here we denoted the identity operation on $\mathcal{S}\left(\mathbb{R}^{d m}, \mathbb{C}^{q^{m}}\right)$ by $\mathbf{1}_{m}$. Therefore one gets

$$
\begin{aligned}
& |R \otimes S(\chi)| \leq C_{R}\|\varrho\|_{K, N} \\
& =C_{R} \sup _{\substack{x_{1}, \cdots, x_{n} \in \mathbb{R}^{d} \\
0 \leq\left|\alpha_{1}\right|, \cdots,\left|\alpha_{n}\right|<K}} \prod_{l=1}^{m}\left(1+\left|x_{l}\right|^{2}\right)^{N / 2} \\
& \times\left|S\left(D^{\alpha_{1} \cdots \alpha_{n}} \otimes \mathbf{1}_{n} \chi\left(x_{1}, \cdots, x_{m}, .\right)\right)\right| \\
& \leq C_{R} \sup _{\substack{x_{1}, \cdots, x_{n} \in \mathbb{R}^{d} \\
0 \leq\left|\alpha_{1}\right|, \cdots,\left|\alpha_{n}\right| \leq K}} \prod_{l=1}^{m}\left(1+\left|x_{l}\right|^{2}\right)^{N / 2}
\end{aligned}
$$

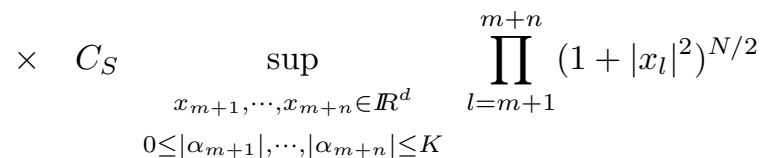

$$
\begin{aligned}
& \times \quad\left|D^{\alpha_{1} \cdots \alpha_{m}} \otimes D^{\alpha_{m+1} \cdots \alpha_{m+n}} \chi\left(x_{1}, \cdots, x_{m+n}\right)\right| \\
& =C_{R} C_{S}\|\chi\|_{K, N}
\end{aligned}
$$

The following theorem gives a sufficient Hilbert space structure condition on the truncated Wightman functions:

Theorem 2.2 Let $K, N \in \mathbb{N}_{0}$ and let $\left\{a_{n}\right\}_{n \in \mathbb{N}}$ be a sequence of positive constants, such that for all $n \in \mathbb{N}$ the truncated n-point Wightman function $\mathbf{W}_{n}^{T}$ fulfills

$$
\left|\mathrm{W}_{n}^{T}(\varphi)\right| \leq a_{n}\|\varphi\|_{K, N}, \quad \forall \varphi \in \mathcal{S}\left(\mathbb{R}^{d n}, \mathbb{C}^{q^{n}}\right) .
$$


Then the corresponding sequence of Wightman functions fulfills the Hilbert space structure condition.

We first prove an auxiliary lemma:

Lemma 2.3 For any sequence of positive constants $\left\{b_{n}\right\}_{n \in \mathbb{N}}$ there exists a sequence of positive constants $\left\{c_{n}\right\}_{n \in \mathbb{N}}$, such that for all $m, n \in \mathbb{N}$ the inequality $b_{m+n} \leq c_{m} c_{n}$ holds.

Proof Let $c_{n}:=\max \left\{\max \left\{b_{j}: 1 \leq j \leq 2 n\right\}, 1\right\}$. Then for all $m, n \in \mathbb{N}$, we have $b_{m+n} \leq \max \left\{c_{m}, c_{n}\right\} \leq c_{m} c_{n}$.

Proof of Theorem 2.2 For $n \in \mathbb{N}$ we define

$$
b_{n}:=\sum_{I \in \mathcal{P}^{(n)}} \prod_{\left\{j_{1}, \cdots, j_{l}\right\} \in I} a_{l} .
$$

For $m, n \in \mathbb{N}, \chi \in \mathcal{S}\left(\mathbb{R}^{d(m+n)}, \mathbb{C}^{q^{(m+n)}}\right)$, we get by inductive use of (9) and Lemma 2.1

$$
\left|\mathrm{W}_{m+n}(\chi)\right| \leq b_{m+n}\|\chi\|_{K, N} .
$$

Now we take $\chi=\varphi^{*} \otimes \eta, \varphi \in \mathcal{S}\left(\mathbb{R}^{d m}, \mathbb{C}^{q^{m}}\right), \eta \in \mathcal{S}\left(\mathbb{R}^{d n}, \mathbb{C}^{q^{n}}\right)$, then by (8) we get

$$
\left|\mathrm{W}_{m+n}\left(\varphi^{*} \otimes \eta\right)\right| \leq b_{m+n}\|\varphi\|_{K, N}\|\eta\|_{K, N} .
$$

On the other hand, by Lemma 2.3 there exists a sequence of positive numbers $\left\{c_{n}\right\}_{n \in \mathbb{N}}$ such that

$$
\left|\mathrm{W}_{m+n}\left(\varphi^{*} \otimes \eta\right)\right| \leq c_{m}\|\varphi\|_{K, N} c_{n}\|\eta\|_{K, N} .
$$

By Vol.IV (see p. 82) of [8] for $n \in \mathbb{N}$ there is a system $\left\{\|\cdot\|_{K, N}^{\prime}\right\}_{K, N \in \mathbb{N}_{0}}$ of Hilbert norms on $\mathcal{S}\left(\mathbb{R}^{d n}, \mathbb{C}^{q^{n}}\right)$ which is equivalent to the system of Schwartz norms $\left\{\|\cdot\|_{K, N}\right\}_{K, N \in \mathbb{N}_{0}}$. Thus, there is a sequence of positive constants $\left\{d_{n}\right\}_{n \in \mathbb{N}}$ such that for the above fixed $K, N \in \mathbb{N}_{0}$ and suitable $K^{\prime}, N^{\prime} \in \mathbb{N}_{0}$ (depending on $K, N$ and $n$ ) we get

$$
\|\varphi\|_{K, N} \leq d_{n}\|\varphi\|_{K^{\prime}, N^{\prime}}^{\prime}, \forall \varphi \in \mathcal{S}\left(\mathbb{R}^{d n}, \mathbb{C}^{q^{n}}\right) .
$$

We now choose Hilbert norms $p_{n}$ on $\mathcal{S}\left(\mathbb{R}^{d n}, \mathbb{C}^{q^{n}}\right)$ as $p_{n}(\cdot):=c_{n} d_{n}\|\cdot\|_{K^{\prime}, N^{\prime}}^{\prime}$. From (10) we immediately get the Hilbert space structure condition

$$
\left|\mathrm{W}_{m+n}\left(\varphi^{*} \otimes \eta\right)\right| \leq p_{m}(\varphi) p_{n}(\eta) .
$$

Since all truncated Wightman functions $\mathrm{W}_{n}^{T}$ are tempered distributions and are therefore continuous with respect to some norm $\|\cdot\|_{K(n), N(n)}$, it is enough to check (9) for $n$ larger than a certain number $m \in \mathbb{N}$ : We may simply put $K^{\prime}:=\max \{K, K(n): n=1, \cdots, m\}, N^{\prime}:=\max \{N, N(n): n=1, \cdots, m\}$ and by $\|\cdot\|_{K^{\prime}, N^{\prime}} \geq\|\cdot\|_{K, N}$ and $\|\cdot\|_{K^{\prime}, N^{\prime}} \geq\|\cdot\|_{K(n), N(n)}, n=1, \cdots, m$, we get (9) for all $n \in \mathbb{N}$ if the numbers $K, N$ are replaced by $K^{\prime}, N^{\prime}$. In particular, we get 
Corollary 2.4 Let $\left\{\mathrm{W}_{n}^{T}\right\}_{n \in \mathbb{N}}$ be a sequence of truncated Wightman distributions. If $\mathrm{W}_{n}^{T}=0$ for all $n$ larger than a certain number $m \in \mathbb{N}$, then the corresponding sequence of Wightman functions fulfills the Hilbert space structure condition.

Since in our models introduced in Section 3, we have explicit formulae for the Fourier transformed truncated Wightman functions rather than for the truncated Wightman functions themselves, we need the following Fourier transformed version of Theorem 2.2:

Corollary 2.5 Let $K, N$ and $\left\{a_{n}\right\}_{n \in \mathbb{N}}$ as in Theorem 2.2. Suppose that $\forall n \in$ $\mathbb{N}$ the Fourier transformed truncated n-point Wightman function $\hat{\mathrm{W}}_{n}^{T}$ fulfills

$$
\left|\hat{\mathrm{W}}_{n}^{T}(\varphi)\right| \leq a_{n}\|\varphi\|_{K, N}, \forall \varphi \in \mathcal{S}\left(\mathbb{R}^{d n}, \mathbb{C}^{q^{n}}\right) .
$$

Then the sequence of Wightman functions fulfills the Hilbert space structure condition.

Proof By the basic fact that $\mathrm{W}_{n}(\varphi)=\hat{\mathrm{W}}_{n}(\hat{\varphi}) \forall \varphi \in \mathcal{S}\left(\mathbb{R}^{d n}, \mathbb{C}^{q^{n}}\right)$ we only have to replace the sequence of Hilbert norms $\left\{p_{n}\right\}_{n \in \mathbb{N}}$ constructed in the proof of Theorem 2.2 by the sequence $\left\{\hat{p}_{n}\right\}_{n \in \mathbb{N}}$ defined as $\hat{p}_{n}:=p_{n} \circ$. Then $\left\{\mathrm{W}_{n}\right\}_{n \in N_{0}}$ fulfills the Hilbert space structure condition with respect to $\left\{\hat{p}_{n}\right\}_{n \in \mathbb{N}}$.

\section{Relativistic fields from convoluted generalized white noise}

Since the work by Nelson[14], the problem of constructing Markovian or reflection positive(see [15] for the notion of reflection positivity) random fields over $\mathbb{R}^{d}$, which are invariant(i.e., homogeneous, stationary) with respect to the Euclidean group, has been looked upon as closely related to the problem of constructing (Bosonic) relativistic quantum fields. In such an approach, the moments of such Euclidean random fields are viewed as Schwinger functions which are the analytic continuation of the vacuum expectation value (Wightman functions) of relativistic quantum fields to purely imaginary time.

In this section, we introduce Wightman functions associated with scalar and vector convoluted generalized white noise Euclidean random fields. Such kind of Euclidean random fields are solutions of certain stochastic partial (pseudo)differential equations of the form $L X=F$ with $F$ a Euclidean generalized white noise and $L$ a suitable invariant (pseudo-)differential operator. In the case where $F$ is a scalar Gaussian white noise and $L=\left(-\Delta+m^{2}\right)^{\alpha}$ with $\alpha \in\left(0, \frac{1}{2}\right]$, the obtained random field $X$ is a generalized free Euclidean scalar quantum field(see e.g. [17]). In the case that $F$ is a quaternionic Gaussian white noise, the solution $X$ of the quaternionic Cauchy-Riemann equation $\partial X=F$ driven by $F$ is the free Euclidean electromagnetic quantum field. If $F$ is non Gaussian, the 
corresponding covariant random fields can be interpreted as Euclidean quantum fields with some nonlinear interactions.

As had been investigated in [2] in the scalar case (see also [5] for an axiomatic result in the vector case), under the condition of non-Gaussian white noise, such Euclidean random fields in general lack the reflection positivity property. However, since the Schwinger functions of such random fields can be explicitly calculated, we can perform the analytic continuation of the Schwinger functions to relativistic Wightman functions "by hand"(see [1, 2, 3, 4] and [9]). Using the properties of Euclidean invariance, symmetry and real-valuedness of the Schwinger functions on one hand, and the Osterwalder-Schrader reconstruction theorem (see [15]) on the other hand, we can obtain that the corresponding Wightman functions satisfy the relativistic postulates of invariance, locality and hermiticity, whereas spectral property and cluster property of the Wightman functions can be verified directly from the derived explicit formulae.

In what follows, we only briefly review these constructions. We refer the reader to $[1,2,3,4]$ and [9] for all details.

\subsection{Scalar models}

Let $\mathcal{S}\left(\mathbb{R}^{d}\right)$ be the Schwartz space of all rapidly decreasing real valued $C^{\infty}$ functions on $\mathbb{R}^{d}$ and $\mathcal{S}^{\prime}\left(\mathbb{R}^{d}\right)$ its topological dual. The dual pairing is denoted by $\langle\cdot, \cdot\rangle$. Let $\mathcal{B}$ be the $\sigma$-algebra generated by all cylinder sets of $\mathcal{S}^{\prime}\left(\mathbb{R}^{d}\right)$. Then $\left(\mathcal{S}^{\prime}\left(\mathbb{R}^{d}\right), \mathcal{B}\right)$ is a standard measurable space.

By the well-known Bochner-Minlos theorem (see e.g. [10] or Vol. IV of [8]), there exists a unique probability measure $P$ on $\left(\mathcal{S}^{\prime}\left(\mathbb{R}^{d}\right), \mathcal{B}\right)$ such that its Fourier transform satisfies

$$
\int_{\mathcal{S}^{\prime}\left(\mathbb{R}^{d}\right)} e^{i<\varphi, \omega>} d P(\omega)=\exp \left\{\int_{\mathbb{R}^{d}} \psi(\varphi(x))\right\}, \varphi \in \mathcal{S}\left(\mathbb{R}^{d}\right)
$$

where $\psi$ is a Lévy-Khinchine function on $\mathbb{R}$ given by

$$
\psi(t)=i a t-\frac{1}{2} \sigma^{2} t^{2}+\int_{\mathbb{R} \backslash\{0\}}\left(e^{i s t}-1-\frac{i s t}{1+s^{2}}\right) d M(s), t \in \mathbb{R}
$$

with $a, \sigma \in \mathbb{R}$ and $M$ is a non-decreasing function satisfying

$$
\int_{\mathbb{R} \backslash\{0\}} \min \left(1, s^{2}\right) d M(s)<\infty .
$$

We call $P$ a generalized white noise measure with Lévy-Khinchine function $\psi$. The associated coordinate process $F: \mathcal{S}\left(\mathbb{R}^{d}\right) \times\left(\mathcal{S}^{\prime}\left(\mathbb{R}^{d}\right), \mathcal{B}, P\right) \rightarrow \mathbb{R}$ defined by

$$
F(\varphi, \omega):=<\varphi, \omega>, \varphi \in \mathcal{S}\left(\mathbb{R}^{d}\right), \omega \in \mathcal{S}^{\prime}\left(\mathbb{R}^{d}\right)
$$

is called a generalized white noise. 
Let $K: \mathbb{R}^{d} \times \mathbb{R}^{d} \rightarrow \mathbb{R}$ be a measurable integral kernel such that

$$
(\mathcal{G} \varphi)(x):=\int_{\mathbb{R}^{d}} K(x, y) \varphi(y) d y, \varphi \in \mathcal{S}\left(\mathbb{R}^{d}\right)
$$

is a linear continuous mapping from $\mathcal{S}\left(\mathbb{R}^{d}\right)$ to itself. Then the conjugate mapping $\tilde{\mathcal{G}}: \mathcal{S}^{\prime}\left(\mathbb{R}^{d}\right) \rightarrow \mathcal{S}^{\prime}\left(\mathbb{R}^{d}\right)$ is a measurable transform from $\left(\mathcal{S}^{\prime}\left(\mathbb{R}^{d}\right), \mathcal{B}\right)$ to itself. Let $P_{K}$ denote the image measure of $P$ under $\tilde{\mathcal{G}}$ :

$$
P_{K}(A):=P\left(\tilde{\mathcal{G}}^{-1} A\right), \quad A \in \mathcal{B} .
$$

Then it is not hard to derive that

$$
\int_{\mathcal{S}^{\prime}\left(\mathbb{R}^{d}\right)} e^{i<\varphi, \omega>} d P_{K}(\omega)=\exp \left\{\int_{\mathbb{R}^{d}} \psi\left(\int_{\mathbb{R}^{d}} K(x, y) \varphi(y) d y\right) d x\right\}
$$

for $\varphi \in \mathcal{S}\left(\mathbb{R}^{d}\right)$. The coordinate process $X: \mathcal{S}\left(\mathbb{R}^{d}\right) \times\left(\mathcal{S}^{\prime}\left(\mathbb{R}^{d}\right), \mathcal{B}, P_{K}\right) \rightarrow \mathbb{R}$ given by

$$
X(\varphi, \omega):=<\varphi, \omega>, \varphi \in \mathcal{S}\left(\mathbb{R}^{d}\right), \omega \in \mathcal{S}^{\prime}\left(\mathbb{R}^{d}\right)
$$

is a random field. Actually, $X$ is precisely $\tilde{\mathcal{G}} F$ defined by $(\tilde{\mathcal{G}} F)(\varphi, \omega):=F(\mathcal{G} \varphi, \omega)$. Moreover, $X$ is a Euclidean field if $K$ is Euclidean invariant. In this case, we can write $K(x, y):=G(x-y)$ for some function $G$ on $\mathbb{R}^{d}$ (with the corresponding invariance property), and for the Euclidean field $X$ we have $X=G * F$, i.e. $X$ is a (Euclidean) convoluted generalized white noise.

Now we assume that all the moments of $M$ in (13) are finite, then $\psi$ is $C^{\infty}$ smooth in a neighborhood of the origin and all the moments of $X$ exist. We define Schwinger functions of $X$ on the topological tensor product $\mathcal{S}^{\otimes n}\left(\mathbb{R}^{d}\right) \cong$ $\mathcal{S}\left(\mathbb{R}^{d n}\right)$ as follows

$$
S_{n}\left(\varphi_{1} \otimes \cdots \otimes \varphi_{n}\right):=\int_{\mathcal{S}^{\prime}\left(\mathbb{R}^{d}\right)} \prod_{j=1}^{n} X\left(\varphi_{j}, \omega\right) d P_{K}(\omega)
$$

Moreover, by using the explicit form of the right hand side of (14), we can calculate the truncated Schwinger functions of the model as follows:

$$
\begin{aligned}
S_{n}^{T}\left(\varphi_{1} \otimes \cdots \otimes \varphi_{n}\right) & :=\left.i^{-n} \frac{\partial^{n}}{\partial \lambda_{1} \cdots \partial \lambda_{n}}\left\{\int_{\mathbb{R}^{d}} \psi\left(\sum_{j=1}^{n} \lambda_{j}\left(G * \varphi_{j}\right)(x)\right) d x\right\}\right|_{\lambda_{1}=\cdots=\lambda_{n}=0} \\
& =c_{n} \int_{\mathbb{R}^{d n}} G^{(n)}\left(x_{1}, \cdots, x_{n}\right) \prod_{j=1}^{n} \varphi_{j}\left(x_{j}\right) \prod_{j=1}^{n} d x_{j}
\end{aligned}
$$

for $\varphi_{1}, \cdots, \varphi_{n} \in \mathcal{S}\left(\mathbb{R}^{d}\right)$ and $n \in \mathbb{N}$, where

$$
c_{1}=a+\int_{\mathbb{R} \backslash\{0\}} \frac{s^{3}}{1+s^{2}} d M(s)
$$




$$
\begin{gathered}
c_{2}=\sigma^{2}+\int_{\mathbb{R} \backslash\{0\}} s^{2} d M(s) \\
c_{n}=\int_{\mathbb{R} \backslash\{0\}} s^{n} d M(s), n \geq 3 \\
G^{(n)}\left(x_{1}, \cdots, x_{n}\right):=\int_{\mathbb{R}^{d}} \prod_{j=1}^{n} G\left(x-x_{j}\right) d x, n \in \mathbb{N} .
\end{gathered}
$$

Furthermore, taking into account that the Schwinger functions can be expressed by partial derivatives of the right hand side of (14) at zero, and using a generalized chain rule, we get the following formula

$$
S_{n}\left(\varphi_{1} \otimes \cdots \otimes \varphi_{n}\right)=\sum_{I \in \mathcal{P}^{n}} \prod_{\left\{j_{1}, \cdots, j_{k}\right\} \in I} S_{k}^{T}\left(\varphi_{j_{1}} \otimes \cdots \otimes \varphi_{j_{k}}\right), n \in \mathbb{N},
$$

which is clearly the same relation as (7).

Taking now $G$ to be the Green function $G_{\alpha}$, say, of the pseudo-differential operator $\left(-\Delta+m_{0}^{2}\right)^{\alpha}$ for the mass $m_{0}>0$ and $\alpha \in\left(0, \frac{1}{2}\right]$, where $\Delta$ is the Laplace operator on $\mathbb{R}^{d}$, namely(e.g. in the sense of Fourier transforms of tempered distributions)

$$
G_{\alpha}(x)=(2 \pi)^{-d} \int_{\mathbf{R}^{d}} \frac{e^{i k x}}{\left(|k|^{2}+m_{0}^{2}\right)^{\alpha}} d k, \quad x \in \mathbf{R}^{d},
$$

then we have Euclidean fields $X=G_{\alpha} * F$ and their Schwinger functions and truncated Schwinger functions as defined above. To perform analytic continuation of $S_{n}^{T}$, we need first to represent $S_{n}^{T}$ in terms of a Laplace transform. In fact, we have (see [1,2] and [9]) a sequence of truncated Wightman functions $\left\{W_{n, \alpha}^{T}\right\}_{n \in \mathbb{N}}$, with the following Laplace transform formula

$$
S_{n}^{T}\left(y_{1}, \cdots, y_{n}\right)=(2 \pi)^{-\frac{d n}{2}} \int_{\mathbf{R}^{d n}} e^{-\sum_{l=1}^{m} k_{l}^{0} y_{l}^{0}+i \vec{k}_{l} \vec{y}_{l}} \hat{W}_{n, \alpha}^{T}\left(k_{1}, \cdots, k_{n}\right) \otimes_{l=1}^{n} d k_{l}
$$

for $y_{1}^{0}<\cdots<y_{n}^{0}$, where $W_{1, \alpha}^{T}:=0$ (we take this for simplicity); $W_{2, \frac{1}{2}}$ is given as $c_{2}$ times the two-point function of the relativistic free field of mass $m_{0}$; for $n \geq 3$ or $n=2$ and $\alpha \in\left(0, \frac{1}{2}\right)$,

$$
\begin{aligned}
\hat{W}_{n, \alpha}^{T}\left(k_{1}, \cdots, k_{n}\right):= & c_{n} 2^{n-1}(2 \pi)^{d}\left\{\sum_{j=1}^{n} \prod_{l=1}^{j-1} \mu_{\alpha}^{-}\left(k_{l}\right) \mu_{\alpha}\left(k_{j}\right) \prod_{l=j+1}^{n} \mu_{\alpha}^{+}\left(k_{l}\right)\right\} \\
& \times \delta\left(\sum_{l=1}^{n} k_{l}\right) .
\end{aligned}
$$


are tempered distributions with

$$
\begin{aligned}
& \mu_{\alpha}^{+}(k):=(2 \pi)^{-d / 2} \sin \pi \alpha 1_{\left\{k^{2}>m_{0}^{2}, k^{0}>0\right\}}(k) \frac{1}{\left(k^{2}-m_{0}^{2}\right)^{\alpha}} \\
& \mu_{\alpha}^{-}(k):=(2 \pi)^{-d / 2} \sin \pi \alpha 1_{\left\{k^{2}>m_{0}^{2}, k^{0}<0\right\}}(k) \frac{1}{\left(k^{2}-m_{0}^{2}\right)^{\alpha}} \\
& \mu_{\alpha}(k):=(2 \pi)^{-d / 2}\left(\cos \pi \alpha 1_{\left\{k^{2}>m_{0}^{2}\right\}}(k)+1_{\left\{k^{2}<m_{0}^{2}\right\}}(k)\right) \frac{1}{\left|k^{2}-m_{0}^{2}\right|^{\alpha}}
\end{aligned}
$$

where $k:=\left(k^{0}, \vec{k}\right) \in \mathbb{R} \times \mathbb{R}^{d-1}$.

By the general property of Laplace transform, $S_{n}^{T}$ can be analytically continued from the purely Euclidean imaginary time to the permuted extended backward tube $T_{p . e .}^{n}$ with the boundary value $W_{n, \alpha}^{T}=\mathcal{F}^{-1} \hat{W}_{n, \alpha}^{T}$ for real (relativistic) time. We then have the following result (see Corollary 7.11 of [2])

Theorem $3.1\left\{W_{n, \alpha}\right\}_{n \in \mathbb{N}}$ defined by $\left\{W_{n, \alpha}^{T}\right\}_{n \in \mathbb{N}}$ via (7) is a sequence of Wightman functions which satisfy Axioms I-IV, the hermiticity condition and the cluster property.

\subsection{Vector models}

Euclidean vector models of quantum fields given by solutions of covariant stochastic partial differential equations with white noise source have been discussed in [3] (see also references therein). We recall here briefly the basic elements, in the case of a four dimensional space-time, identified, in its Euclidean version, with the vector space of quaternions(this identification permitting to write the basic stochastic partial differential equation in a simple form). Thus, let $\mathbb{H}$ be the skew field of all quaternions with $\{\mathbf{1}, \mathbf{i}, \mathbf{j}, \mathbf{k}\}$ its canonical basis. Let $\mathcal{S}\left(\mathbb{R}^{4}, \mathbb{H}\right)$ denote the Schwartz space of all rapidly decreasing functions from $\mathbb{R}^{4}$ to $\mathbb{H}$ and $\mathcal{S}^{\prime}\left(\mathbb{R}^{4}, \mathbb{H}\right)$ its topological dual. The dual pairing is denoted by $\langle\cdot, \cdot\rangle$.

By the known Bochner-Minlos theorem (Vol. IV of [8]), there exists a unique probability measure $P$ on the standard measurable space $\left.\left(\mathcal{S}^{\prime}\left(\mathbb{R}^{4}\right), \mathbb{H}\right), \mathcal{B}\right)$, where $\mathcal{B}$ is the $\sigma$-algebra generated by all cylinder sets of $\mathcal{S}^{\prime}\left(\mathbb{R}^{4}, \mathbb{H}\right)$, with the following Fourier transform

$$
\int_{\mathcal{S}^{\prime}\left(\mathbb{R}^{4}, \mathbb{H}\right)} e^{i<\varphi, \omega>} d P(\omega)=\exp \left\{\int_{\mathbb{R}^{4}} \psi(\varphi(x)) d x\right\}, \quad \varphi \in \mathcal{S}\left(\mathbb{R}^{4}, \mathbb{H}\right)
$$

where $\psi$ is a Lévy-Khinchine function on $\mathbb{H}$ given by

$$
\begin{aligned}
\psi(x)= & i \beta x^{0}-\frac{1}{2} \sigma_{0} x^{0^{2}}-\frac{1}{2} \sigma|\vec{x}|^{2} \\
& -\int_{\mathbb{H} \backslash\{0\}}\left(1+i<x, y>_{E} 1_{(0,1)}(|y|)-e^{i<x, y>_{E}}\right) \nu(d y)
\end{aligned}
$$


with the condition that $\psi(x)=O\left(|x|^{\frac{4}{3}+\epsilon}\right)$ as $x \rightarrow 0$, where $x:=x^{0} \mathbf{1}-x^{1} \mathbf{i}-$ $x^{2} \mathbf{j}-x^{3} \mathbf{k},\left(x^{0}, x^{1}, x^{2}, x^{3}\right) \in \mathbb{R}^{4}, \vec{x}:=x-\mathbf{1} x^{0}, \beta \in \mathbb{R}, \sigma_{0}, \sigma \in(0, \infty),|x|$ denotes the Euclidean norm of $x \in \mathbb{H}$ and $\nu$ is a Lévy measure on $\mathbb{H}$ supported by the centre of $\mathbb{H} \backslash\{0\}$ (see [3]).

In the same way as in Subsection 3.1, we can define the associated coordinate process $F: \mathcal{S}\left(\mathbb{R}^{4}, \mathbb{H}\right) \times\left(\mathcal{S}^{\prime}\left(\mathbb{R}^{4}, \mathbb{H}\right), \mathcal{B}, P\right) \rightarrow \mathbb{R}$ by

$$
F(\varphi, \omega):=<\varphi, \omega>, \varphi \in \mathcal{S}\left(\mathbb{R}^{4}, \mathbb{H}\right), \omega \in \mathcal{S}^{\prime}\left(\mathbb{R}^{4}, \mathbb{H}\right) .
$$

We call $F$ a $\mathbb{H}$-valued generalized white noise.

The covariant vector random fields were constructed in [3] as solutions of the inhomogeneous quaternionic Cauchy-Riemann equation $\partial X=F$ over $\mathbb{H}$, where $\partial$ is the quaternionic Cauchy- Riemann operator defined by

$$
\partial:=\mathbf{1} \frac{\partial}{\partial x^{0}}-\mathbf{i} \frac{\partial}{\partial x^{1}}-\mathbf{j} \frac{\partial}{\partial x^{2}}-\mathbf{k} \frac{\partial}{\partial x^{3}} .
$$

The conjugate operator $\bar{\partial}$ of $\partial$ is given by

$$
\bar{\partial}:=\mathbf{1} \frac{\partial}{\partial x^{0}}+\mathbf{i} \frac{\partial}{\partial x^{1}}+\mathbf{j} \frac{\partial}{\partial x^{2}}+\mathbf{k} \frac{\partial}{\partial x^{3}}
$$

and the Laplace operator is defined by $\Delta_{\mathbb{H}}:=\partial \bar{\partial}=\bar{\partial} \partial$. The Green function for $-\Delta_{\mathbb{H}}$ is given explicitly by

$$
g(x)=\frac{1}{4 \pi^{2}|x|^{2}}, x \in \mathbb{H} \backslash\{0\} .
$$

Then the equation $\partial X=F$ is solved by the convolution $X=(-\bar{\partial} g) * F$ which is the coordinate process associated to the probability measure $P_{X}$ on $\left(\mathcal{S}^{\prime}\left(\mathbb{R}^{4}, \mathbb{H}\right), \mathcal{B}\right)$ determined by the following Fourier transform

$$
\int_{\mathcal{S}^{\prime}\left(\mathbb{R}^{4}, \mathbb{H}\right)} e^{i<\varphi, \omega>} d P_{X}(\omega)=\exp \left\{\int_{\mathbb{R}^{4}} \psi((g * \partial \varphi)(x)) d x\right\}, \varphi \in \mathcal{S}\left(\mathbb{R}^{4}, \mathbb{H}\right) .
$$

Similarly to the scalar case in subsection 3.1, under the assumption that $\nu$ has moments of all orders large than one, $\psi$ is $C^{\infty}$-smooth in a neighborhood of $0 \in \mathbb{H}$. The Schwinger functions $S_{n}, n \in \mathbb{N}$ and the truncated Schwinger functions $S_{n}^{T}, n \in \mathbb{N}$ of $X$ can be constructed explicitly as follows

$$
S_{n}\left(\varphi_{1} \otimes \cdots \otimes \varphi_{n}\right):=\int_{\mathcal{S}^{\prime}\left(\mathbb{R}^{4}, \mathbb{H}\right)} \prod_{j=1}^{n} X\left(\varphi_{j}, \omega\right) d P_{X}(\omega), n \in \mathbb{N}
$$

and

$$
\begin{aligned}
S_{n}^{T}\left(\varphi_{1} \otimes \cdots \otimes \varphi_{n}\right): & :=\left.i^{-n} \frac{\partial^{n}}{\partial \lambda_{1} \cdots \partial \lambda_{n}}\left\{\int_{\mathbb{R}^{4}} \psi\left(\sum_{j=1}^{n} \lambda_{j}\left(g * \partial \varphi_{j}\right)(x)\right) d x\right\}\right|_{\lambda_{1}=\cdots=\lambda_{n}=0} \\
= & \begin{cases}\text { constant }, & n=1 \\
<c_{0} \operatorname{div} \varphi_{1} \otimes \operatorname{div} \varphi_{2}+c D^{E}\left(\varphi_{1} \otimes \varphi_{2}\right), g^{(2)}>, & n=2 \\
<\mathcal{E}^{n} \varphi_{1} \otimes \cdots \otimes \varphi_{n}, g^{(n)}>, & n \geq 3\end{cases}
\end{aligned}
$$


for $\varphi_{1}, \cdots, \varphi_{n} \in \mathcal{S}\left(\mathbb{R}^{4}, \mathbb{H}\right)$, where

$$
g^{(n)}(\underline{y})=\left\{\begin{array}{l}
-\frac{1}{8 \pi} \ln \left|y_{1}-y_{2}\right|, n=2 \\
\int_{\mathbb{R}^{4}} \prod_{j=1}^{n} g\left(x-y_{j}\right) d x, n \geq 3 .
\end{array}\right.
$$

for $\underline{y}=\left(y_{1}, \cdots, y_{n}\right) \in\left(\mathbb{R}^{4}\right)_{\neq}^{n}:=\left\{\underline{y} \in\left(\mathbb{R}^{4}\right)^{n}: y_{j} \neq y_{l}\right.$ if $\left.j \neq l\right\}$,

$$
\begin{gathered}
\mathcal{E}^{n}:=\sum_{l=0, l: \text { even }}^{n} c_{l}^{n} \mathcal{E}_{l}^{n}, \\
\left\{\begin{array}{l}
c_{0}:=\sigma_{0}+\int_{\mathbb{I H} \backslash\{0\}} x^{0^{2}} \nu(d x), \\
c:=\sigma+\frac{1}{3} \int_{\mathbb{I H} \backslash\{0\}}|\vec{x}|^{2} \nu(d x), \\
c_{l}^{n}:=\left(\begin{array}{c}
n \\
l
\end{array}\right) \frac{1}{l+1} \int_{\mathbb{I H} \backslash\{0\}} x^{0^{n-l}|\vec{x}|^{l} \nu(d x), n \geq 3,0 \leq l \leq n,} \\
\mathcal{E}_{l}^{n}:=\operatorname{Sym}(\underbrace{\operatorname{div} \otimes \cdots \otimes \operatorname{div}}_{n-l} \otimes \underbrace{D^{E} \otimes \cdots \otimes D^{E}}_{\frac{l}{2}}),
\end{array}\right.
\end{gathered}
$$

and $D^{E}: \mathcal{S}\left(\mathbb{R}^{4} \times \mathbb{R}^{4}, \mathbb{H} \times \mathbb{H}\right) \rightarrow \mathcal{S}\left(\mathbb{R}^{4} \times \mathbb{R}^{4}, \mathbb{R}\right)$ is a linear partial differential operator on $\mathbb{R}^{4} \times \mathbb{R}^{4}$ which is of first order with respect to each variable $x_{1}, x_{2} \in$ $\mathbb{R}^{4}$.

The analytic continuation of $\left\{S_{n}^{T}\right\}_{n \in \mathbb{N}}$ from the imaginary Euclidean time to the real relativistic time performed in [3] (to which we refer for details) yields a sequence of truncated Wightman functions $\left\{W_{n}^{T}\right\}_{n \in \mathbb{N}}$. In fact, each $g^{(n)}$ defined by (19) has a holomorphic extension $G^{(n)}$ defined on the permuted extended backward tube $T_{p . e .}^{n}$. For $n \geq 3$, it is defined as follows:

$$
G^{(n)}(\underline{z}):=<e(\underline{z}, \cdot), M_{0}^{n}+\sum_{j=1}^{n-1}\left(\frac{\partial}{\partial k_{j+1}^{0}}-\frac{\partial}{\partial k_{j}^{0}}\right) M_{j}^{n}+M_{n}^{n}>, \underline{z} \in T_{p . e .}^{n}
$$

where

$$
e(\underline{z}, \underline{k}):=(2 \pi)^{-2 n} \exp \left\{i \sum_{j=1}^{n}<z_{j}, k_{j}>_{E}\right\}
$$

and $\left\{M_{j}^{n}: 0 \leq j \leq n\right\}$ are measures defined on the space $\mathbb{R}^{4 n}$ (see [3]). This can be verified by writing $g^{(n)}$ as the Laplace transform (c.f. equation (17)) of the following tempered distribution

$$
M_{0}^{n}+\sum_{j=1}^{n-1}\left(\frac{\partial}{\partial k_{j+1}^{0}}-\frac{\partial}{\partial k_{j}^{0}}\right) M_{j}^{n}+M_{n}^{n} .
$$

In what follows, we will give a representation of $\left\{M_{j}^{n}: 0 \leq j \leq n\right\}$, which is different from the one given in [3], for later use in Section 4, which can be derived from the argument in Subsection 7.4 of [2] in the case $m_{0}=0$ : 


$$
\begin{aligned}
& M_{0}^{n}=(2 \pi)^{3-n} \frac{1}{2\left|\vec{k}_{1}\right|\left(k_{1}^{0}-\left|\vec{k}_{1}\right|\right)} \prod_{l=2}^{n} \delta_{0}^{+}\left(k_{l}\right) \delta\left(\sum_{l=1}^{n} k_{l}\right) ; \\
& M_{n}^{n}=(2 \pi)^{3-n} \frac{1}{2\left|\vec{k}_{n}\right|\left(k_{n}^{0}+\left|\vec{k}_{n}\right|\right)} \prod_{l=1}^{n-1} \delta_{0}^{-}\left(k_{l}\right) \delta\left(\sum_{l=1}^{n} k_{l}\right) ; \\
& M_{j}^{n}(\varphi)= \\
&(2 \pi)^{3-n} \int_{0}^{1}<\prod_{l=1}^{j-1} \delta_{0}^{-}\left(k_{l}\right) \frac{\delta\left(k_{j}^{0}-\tilde{k}_{j}^{0}(s)\right)}{4 \mid \overrightarrow{k_{j}|| k_{j+1} \mid}} \prod_{l=j+2}^{n} \delta_{0}^{+}\left(k_{l}\right) \delta\left(\sum_{l=1}^{n} k_{l}\right), \varphi>d s,
\end{aligned}
$$

for $1 \leq j \leq n-1, \varphi \in \mathcal{S}\left(\mathbb{R}^{4 n}\right)$, where

$$
\begin{aligned}
\delta_{0}^{+}\left(k_{l}\right) & =1_{\left\{k_{l}^{0}>0\right\}}\left(k_{l}^{0}\right) \delta\left(k_{l}^{2}\right), \\
\delta_{0}^{-}\left(k_{l}\right) & =1_{\left\{k_{l}^{0}<0\right\}}\left(k_{l}^{0}\right) \delta\left(k_{l}^{2}\right), \\
\tilde{k}_{j}^{0}(s) & =\tilde{k}_{j}^{0}\left(k_{1}^{0}, \cdots, k_{j-1}^{0}, \vec{k}_{j}, \vec{k}_{j+1}, k_{j+1}^{0}, \cdots, k_{n}^{0}, s\right) \\
& :=-\left\{\left(-\sum_{l=1}^{j-1} k_{l}^{0}+\omega_{j}\right) s+\left(\omega_{j+1}+\sum_{l=j+2}^{n} k_{l}^{0}\right)(1-s)+\sum_{l=1}^{j-1} k_{l}^{0}\right\} .
\end{aligned}
$$

For $n \geq 3$, let $G_{n}$ denote the boundary value of $G^{(n)}$ (under the limit of the purely real time) in the backward tube $T^{n}$. For the case that $n=2, G_{2}$ can be calculated by using a different method. Since here we do not need an explicit formula for $W_{2}^{T}$, we refer to [2] for this calculation. The corresponding truncated Wightman functions $\left\{W_{n}^{T}\right\}_{n \in \mathbb{N}}$ over Minkowski space $M_{4}$ are then given as follows

$$
W_{n}^{T}\left(\varphi_{1} \otimes \cdots \otimes \varphi_{n}\right) \begin{cases}\equiv \text { constant }, & n=1 \\ :=<c_{0} \operatorname{div} \varphi_{1} \otimes \operatorname{div} \varphi_{2}+c D^{M}\left(\varphi_{1} \otimes \varphi_{2}\right), G_{2}>, & n=2 \\ :=<\mathcal{L}^{n} \varphi_{1} \otimes \cdots \otimes \varphi_{n}, G_{n}>, & n \geq 3\end{cases}
$$

for $\varphi_{1}, \cdots, \varphi_{n} \in \mathcal{S}\left(\mathbb{R}^{4}, \mathbb{H}\right)$, where $D^{M}$ is a linear partial differential operator on $\mathbb{R}^{4} \times \mathbb{R}^{4}$ which is obtained as an analytic continuation of $D^{E}$ and hence it is of first order with respect to each variable $x_{1}, x_{2} \in \mathbb{R}^{4}$,

$$
\mathcal{L}^{n}:=\sum_{l=0, l: \text { even }}^{n} c_{l}^{n} \mathcal{L}_{l}^{n}
$$

with

$$
\mathcal{L}_{l}^{n}:=\operatorname{Sym}(\underbrace{\operatorname{div} \otimes \cdots \otimes \operatorname{div}}_{n-l} \otimes \underbrace{D^{M} \otimes \cdots \otimes D^{M}}_{\frac{l}{2}}) .
$$


We notice that $\mathcal{L}_{l}^{n}$ is also a linear partial differential operator on $\underbrace{\mathbb{R}^{4} \times \cdots \times \mathbb{R}^{4}}_{n}$ which is of first order with respect to every variable $x_{1}, \cdots, x_{n} \in \mathbb{R}^{4}$.

We then have the following result from Theorem 4.21 of [3] (cf. Theorem 4.5 and Corollary 4.7 of [2] for the cluster property):

Theorem $3.2\left\{W_{n}\right\}_{n \in \mathbb{N}}$, as defined by $\left\{W_{n}^{T}\right\}_{n \in \mathbb{N}}$ via (7), is a sequence of Wightman functions which satisfy Axioms I-IV, the hermiticity condition and the cluster property.

\section{Verification of the Hilbert space structure con- dition for the models}

In this section we prove that the truncated Wightman functions of the scalar models as well as the vector models in Section 3 fulfill the requirements of Corollary 2.5, which further implies that the Wightman functions of both the scalar and vector models in Section 3 satisfy Axiom V. Thus, we prove the following result:

Theorem 4.1 The Wightman functions obtained in Section 3 for the scalar and the vector models(over the d-dimensional resp. 4-dimensional Minkowski space-time) fulfill the modified Wightman axioms I-V (of Morchio and Strocchi).

In particular, for each such model there is a Hilbert space $(\mathcal{H},(\cdot, \cdot))$, a continuous and self adjoint metric operator $T$ on $\mathcal{H}$ fulfilling $T^{2}=1$ and local $T$-symmetric field operators $\phi(f)$ defined on a common dense domain $\mathcal{D} \subset \mathcal{H}$ for $f \in \mathcal{S}\left(\mathbb{R}^{d}, \mathbb{C}\right), \mathcal{S}\left(\mathbb{R}^{4}, \mathbb{C}^{4}\right)$ respectively, such that equation (3) holds. Furthermore, we have a $T$-unitary representation $\mathcal{U}$ of (the proper orthochronous Poincaré group over $\mathbb{R}^{d}$ resp. $\left.\mathbb{R}^{4}\right) \mathcal{P}_{+}^{\uparrow}$ on the dense domain $\mathcal{D} \subset \mathcal{H}$, where the transformation law of the fields $\phi$ under $\mathcal{U}$ is given by (4) and $\mathcal{U}$ fulfills the spectral condition as given in the equation (5).

The second part of theorem 4.1 by the results of Section 1 immediately follows from the Axioms I-V.

Although there is a lot of similarity in the methods applied to the scalar and the vector model, the origin of the technical difficulties in the proof of Axiom $\mathrm{V}$ in both cases is quite different:

In the scalar case the Källen-Lehmann representation of the Green functions $G_{\alpha}$ by infinite measures [2] leads to singularities of the Fourier transformed Wightman distributions near the mass shell of the lowest mass. These singularities for $0<\alpha \leq \frac{1}{2}$ turn out to be locally integrable independently of the dimension $d$ of the underlying space-time.

In the vector case we restricted ourselves to a special Green function, such that the above mentioned singularities do not arise. But in this case we have to 
overcome the problems caused by the fact that the fields have mass zero, leading to singularities at the bottom of the light cone. These singularities are however locally integrable, since we have specialized to the sufficiently large (physical) space-time dimension 4 .

\subsection{Proof of Theorem 4.1 for the scalar models}

By the argument given in Section 2, it suffices to check equation (11) for $n \geq 3$, $K=0$ and $N=2 d$. Using the explicit formulae of $\hat{W}_{n, \alpha}^{T}$ for $\alpha \in\left(0, \frac{1}{2}\right]$ with $m_{0}>0$, we get that for $\varphi \in \mathcal{S}\left(\mathbb{R}^{d n}, \mathbb{C}\right)$

$$
\begin{aligned}
\left|\hat{W}_{n, \alpha}^{T}(\varphi)\right| & \leq n c_{n} 2^{n-1}(2 \pi)^{d-\frac{d n}{2}} \int_{\mathbb{R}^{d(n-1)}} \prod_{l=2}^{n} \frac{\left|k_{l}^{2}-m_{0}^{2}\right|^{-\alpha}}{\left(1+\left|k_{l}\right|^{2}\right)^{d}} \\
& \times\left|\left(\sum_{l=2}^{n} k_{l}\right)^{2}-m_{0}^{2}\right|^{-\alpha} \bigotimes_{l=2}^{n} d k_{l}\|\varphi\|_{0,2 d} .
\end{aligned}
$$

It remains to show that the integral on the RHS is finite. Noticing that $(1+$ $\left.\left|k_{l}\right|^{2}\right)^{-d} \leq\left(1+k_{l}^{0^{2}}\right)^{-1}\left(1+\left|\vec{k}_{l}\right|^{2}\right)^{1-d}$, the above integral can be estimated by the following expression:

$$
\begin{aligned}
& \left(\int_{\mathbb{R}^{d-1}} \frac{d \vec{k}}{\left(1+|\vec{k}|^{2}\right)^{d-1}}\right)^{n-1}\left(\sup _{\vec{k} \in \mathbb{R}^{d-1}} \int_{\mathbb{R}} \frac{\left|k^{2}-m_{0}^{2}\right|^{-\alpha}}{\left(1+k^{0^{2}}\right)} d k^{0}\right)^{n-3} \\
\times \quad & \sup _{\substack{\vec{k}_{2}, \vec{k}_{3} \in \mathbb{R}^{d-1} \\
k_{4}, \cdots, k_{n} \in \mathbb{R}^{d}}} \int_{\mathbb{R}^{2}} \frac{\left.\mid\left(\sum_{l=2}^{n} k_{l}\right)^{2}-m_{0}^{2}\right)\left.\left(k_{2}^{2}-m_{0}^{2}\right)\left(k_{3}^{2}-m_{0}^{2}\right)\right|^{-\alpha}}{\left(1+k_{2}^{0^{2}}\right)\left(1+k_{3}^{0^{2}}\right)} d k_{2}^{0} d k_{3}^{0} .
\end{aligned}
$$

Clearly the first factor in $(22)$ is finite. It remains to show that the remaining two factors are also finite.

Since $\left|k^{2}-m_{0}^{2}\right|^{-\alpha}=\left|k_{l}^{0}+\omega\right|^{-\alpha}\left|k_{l}^{0}-\omega\right|^{-\alpha}$, where $\omega=\left(|\vec{k}|^{2}+m_{0}^{2}\right)^{\frac{1}{2}}$ and therefore $\omega \geq m_{0}$, we get that

$$
\left|k^{2}-m_{0}^{2}\right|^{-\alpha} \leq\left|m_{0}\left(k^{0}+\omega\right)\right|^{-\alpha}+\left|m_{0}\left(k^{0}-\omega\right)\right|^{-\alpha}
$$

We set $\omega_{1}:=\left(\left|\sum_{l=2}^{n} \vec{k}_{l}\right|^{2}+m_{0}^{2}\right)^{\frac{1}{2}}, \omega_{l}:=\left(\left|\vec{k}_{l}\right|^{2}+m_{0}^{2}\right)^{\frac{1}{2}}$ for $l=2, \cdots, n$.By $(23)$ the integral in the second factor in equation $(22)$ can be estimated by $m_{0}^{-\alpha}$ times two integrals of the following kind

$$
\begin{aligned}
\int_{\mathbb{R}} \frac{\left|k^{0} \pm \omega\right|^{-\alpha}}{1+k^{0^{2}}} d k^{0} & =\int_{\left\{\left|k^{0} \pm \omega\right|<1\right\}} \frac{\left|k^{0} \pm \omega\right|^{-\alpha}}{1+k^{0^{2}}} d k^{0}+\int_{\left\{\left|k^{0} \pm \omega\right|>1\right\}} \frac{\left|k^{0} \pm \omega\right|^{-\alpha}}{1+k^{0^{2}}} d k^{0} \\
& <\frac{2}{1-\alpha}+\int_{\mathbb{R}} \frac{1}{1+k^{0^{2}}} d k^{0}<\infty
\end{aligned}
$$


Here the latter estimate is independent of $\vec{k} \in \mathbb{R}^{d-1}$. Consequently the second factor in (22) is also finite. It remains to deal with the third factor.

Again by (23) the integral in the third factor of (22) can be dominated by $m_{0}^{-\alpha}$ times eight integrals of the type

$$
\int_{\mathbb{R}^{2}} \frac{\left.\mid\left(\sum_{l=2}^{n} k_{l}^{0}\right) \pm \omega_{1}\right)\left.\left(k_{2}^{0} \pm \omega_{2}\right)\left(k_{3}^{0} \pm \omega_{3}\right)\right|^{-\alpha}}{\left(1+k_{2}^{0^{2}}\right)\left(1+{k_{3}^{0}}^{2}\right)} d k_{2}^{0} d k_{3}^{0} .
$$

Therefore, to prove that the third factor in (22) is finite it is sufficient to show that

$$
\sup _{a, b, c \in \mathbb{R}} \int_{\mathbb{R}^{2}} \frac{|x y(x+y+c)|^{-\alpha}}{\left(1+(x+a)^{2}\right)\left(1+(y+b)^{2}\right)} d x d y<\infty .
$$

To prove (24), we set $t:=y+c$, then we get

$$
\int_{\mathbb{R}} \frac{|x(x+t)|^{-\alpha}}{1+(x+a)^{2}} d x=\int_{\mathbb{R}} \frac{\left|\left(x^{\prime}+\frac{t}{2}\right)\left(x^{\prime}-\frac{t}{2}\right)\right|^{-\alpha}}{1+\left(x^{\prime}-\frac{t}{2}+a\right)^{2}} d x^{\prime} .
$$

For the case that $|t|>2$, the RHS of (25) is smaller than

$$
2 \int_{0}^{\infty} \frac{\left|x^{\prime}-\frac{|t|}{2}\right|^{-\alpha}}{1+\left(x^{\prime}-\frac{|t|}{2}\right)^{2}} d x^{\prime}<2 \int_{\mathbb{R}} \frac{\left|x^{\prime \prime}\right|^{-\alpha}}{1+x^{\prime 2}} d x^{\prime \prime}<\infty
$$

independently of $a \in \mathbb{R}$ and the value of $|t|>2$. We now let $0<|t| \leq 2$. In this case the RHS of (25) independently of $a \in \mathbb{R}$ is smaller than

$$
\int_{-2}^{2}\left|\left(x^{\prime}+\frac{t}{2}\right)\left(x^{\prime}-\frac{t}{2}\right)\right|^{-\alpha} d x^{\prime}+\int_{\mathbb{R}} \frac{1}{1+x^{\prime \prime 2}} d x^{\prime \prime} .
$$

Here the second integral is finite. For any $\gamma \in\left(0, \frac{1}{2}\right)$ the first integral can be further estimated by

$$
2^{1-\gamma}|t|^{-\gamma} \int_{0}^{2}\left|x^{\prime}-\frac{|t|}{2}\right|^{-2 \alpha+\gamma} d x^{\prime} \leq 2^{1-\gamma}|t|^{-\gamma} \int_{-1}^{2}\left|x^{\prime \prime}\right|^{-2 \alpha+\gamma} d x^{\prime \prime}
$$

Since $2 \alpha-\gamma<1$, the integral on the RHS of the above inequality is finite and thus the RHS of (25) is smaller than

$$
C_{1}+C_{2}|t|^{-\gamma}
$$

for sufficiently large constants $C_{1}, C_{2}>0$, which can be chosen independently of the parameter $a \in \mathbb{R}$.

We can therefore estimate the left hand side(LHS) of (24) by

$$
\begin{aligned}
& \sup _{b, c \in \mathbb{R}} \int_{\mathbb{R}} \frac{\left(C_{1}+C_{2}|y-c|^{-\gamma}\right)|y|^{-\alpha}}{1+(y+b)^{2}} d y \\
\leq & C_{1} \sup _{b \in \mathbb{R}} \int_{\mathbb{R}} \frac{|y|^{-\alpha}}{1+(y+b)^{2}} d y+C_{2} \sup _{b, c \in \mathbb{R}} \int_{\mathbb{R}} \frac{|y+c|^{-\alpha-\gamma}+|y|^{-\alpha-\gamma}}{1+(y+b)^{2}} d y .
\end{aligned}
$$


Here the first integral on the RHS of the above inequality is smaller than

$$
\frac{2}{1-\alpha}+\int_{\mathbb{R}} \frac{1}{1+y^{\prime 2}} d y^{\prime}<\infty
$$

and this estimate is independent of $b \in \mathbb{R}$. The second one is dominated by the following constant

$$
\frac{4}{1-\alpha-\gamma}+2 \int_{\mathbb{R}} \frac{1}{1+y^{\prime 2}} d y^{\prime}
$$

which is independent of $b, c \in \mathbb{R}$ and is finite since $\alpha+\gamma<1$.

Thus we have established (24), which was the missing step in the proof of the truncated Hilbert space structure condition (11).

\subsection{Proof of Theorem 4.1 for the vector models}

As in the preceding subsection we want to prove that the requirements of Corollary 2.5 are fulfilled by the Fourier transformed Wightman functions $W_{n}^{T}$ of the vector models described in Section 3.

To this aim, we denote the Fourier transform of the partial differential operators $\mathcal{L}^{n}$ by $\mathcal{M}^{n} . \mathcal{M}^{n}$ is a tensor valued multiplication operator mapping $\mathcal{S}\left(\mathbb{R}^{4 n}, \mathbb{C}^{4^{n}}\right)$ to $\mathcal{S}\left(\mathbb{R}^{4 n}, \mathbb{C}\right)$. Since $\mathcal{L}^{n}$ is a first order partial differential operator in the variables $x_{1}, \cdots, x_{n} \in \mathbb{R}^{4}$, each component of $\mathcal{M}^{n}$ is a polynomial of degree 1 in each of the variables $k_{1}, \cdots, k_{n}$, which are conjugated to $x_{1}, \cdots, x_{n}$ under the Fourier transform. Thus, for $K, N \in \mathbb{N}_{0}$ there exists a constant $C_{1}>0$, such that

$$
\left\|\mathcal{M}^{n} \varphi\right\|_{K, N} \leq C_{1}\|\varphi\|_{K, N+1}, \forall \varphi \in \mathcal{S}\left(\mathbb{R}^{4 n}, \mathbb{C}^{4^{n}}\right) .
$$

By application of the Leibniz rule and the above estimate, we get that there exists a constant $C_{2}>0$, such that also the following inequality holds

$$
\left\|\left(\frac{\partial}{\partial k_{j}^{0}}-\frac{\partial}{\partial k_{j+1}^{0}}\right) \mathcal{M}^{n} \varphi\right\|_{K, N} \leq C_{2}\|\varphi\|_{K+1, N+1}, \forall \varphi \in \mathcal{S}\left(\mathbb{R}^{4 n}, \mathbb{C}^{4^{n}}\right),
$$

for $j=1, \cdots, n-1$. Now let again $n \geq 3$ and $\varphi \in \mathcal{S}\left(\mathbb{R}^{4 n}, \mathbb{C}^{4^{n}}\right)$. From (21) we get

$$
\begin{aligned}
& \hat{W}_{n}^{T}(\varphi)=<\hat{G}_{n}, \mathcal{M}^{n} \varphi> \\
& \quad=<M_{0}^{n}, \mathcal{M}^{n} \varphi>+\sum_{j=1}^{n-1}<M_{j}^{n},\left(\frac{\partial}{\partial k_{j}^{0}}-\frac{\partial}{\partial k_{j+1}^{0}}\right) \mathcal{M}^{n} \varphi>+<M_{n}^{n}, \mathcal{M}^{n} \varphi>.
\end{aligned}
$$

Taking into account the inequalities (26) and (27) we get that

$$
\left|\hat{W}_{n}^{T}(\varphi)\right| \leq a_{n}\|\varphi\|_{K+1, N+1}, \forall \varphi \in \mathcal{S}\left(\mathbb{R}^{4 n}, \mathbb{C}^{4^{n}}\right)
$$


for the constant $a_{n}:=\max \left\{C_{1}, C_{2}\right\} \sum_{j=0}^{n} C_{j}^{n}$, if the measures $M_{j}^{n}, j=1, \cdots, n$ fulfill the conditions

$$
\left|M_{j}^{n}(\varphi)\right| \leq C_{j}^{n}\|\varphi\|_{K, N} \forall \varphi \in \mathcal{S}\left(\mathbb{R}^{4 n}, \mathbb{C}^{4^{n}}\right)
$$

for sufficiently large constants $C_{j}^{n}>0$. Thus, if we can choose $K, N \in \mathbb{N}_{0}$ in (28) independently of $n, j$, the truncated Hilbert space condition of Corollary 2.5 holds.

Let $K=0, N=3$. We first prove (28) for $j=0$ : By (20) we get that

$$
\begin{aligned}
\left|M_{0}^{n}(\varphi)\right| \leq & (2 \pi)^{3-n} 2^{-n} \int_{\mathbb{R}^{3 n-3}} \frac{\left|\vec{k}_{1}+\cdots+\vec{k}_{n}\right|^{-1}}{\left|\vec{k}_{2}\right|+\cdots+\left|\vec{k}_{n}\right|+\left|\vec{k}_{2}+\cdots+\vec{k}_{n}\right|} \\
& \times \prod_{l=2}^{n} \frac{\left|\vec{k}_{l}\right|^{-1}}{\left(1+\left|\vec{k}_{l}\right|^{2}\right)^{3 / 2}} \bigotimes_{l=2}^{n} d \vec{k}_{l}\|\varphi\|_{0,3}
\end{aligned}
$$

We have to show that the integral on the RHS is finite. Since $\left|\vec{k}_{2}\right|+\cdots+\left|\vec{k}_{n}\right|+$ $\left|\vec{k}_{2}+\cdots+\vec{k}_{n}\right| \geq\left|\vec{k}_{3}\right|$ the integral is smaller than the following expression:

$$
\begin{array}{r}
\left(\int_{\mathbb{R}^{3}} \frac{|\vec{k}|^{-1}}{\left(1+|\vec{k}|^{2}\right)^{3 / 2}} d \vec{k}\right)^{n-3}\left(\int_{\mathbb{R}^{3}} \frac{|\vec{k}|^{-2}}{\left(1+|\vec{k}|^{2}\right)^{3 / 2}} d \vec{k}\right) \\
\times\left(\sup _{\vec{a} \in \mathbb{R}^{3}} \int_{\mathbb{R}^{3}} \frac{|\vec{k}+\vec{a}|^{-1}|\vec{k}|^{-1}}{\left(1+|\vec{k}|^{2}\right)^{3 / 2}} d \vec{k}\right) .
\end{array}
$$

Let us consider the first two factors, i.e. we let $\gamma=1,2$ and calculate

$$
\int_{\mathbb{R}^{3}} \frac{|\vec{k}|^{-\gamma}}{\left(1+|\vec{k}|^{2}\right)^{3 / 2}} d \vec{k}=4 \pi \int_{0}^{\infty} \frac{\lambda^{2-\gamma} d \lambda}{\left(1+\lambda^{2}\right)^{3 / 2}}<\infty
$$

since $2-\gamma \geq 0$ and $3-2+\gamma>1$. It remains to show that also the third factor in (29) is finite. For the moment we fix $\vec{a} \in \mathbb{R}^{3}$ and choose orthogonal coordinates, such that $\vec{a}=(a, 0,0)$. Let $\lambda:=\left(k^{2}+k^{3^{2}}\right)^{\frac{1}{2}}$. Using Fubini's theorem we get that the integral in the last factor is smaller than

$$
2 \pi \int_{0}^{\infty} \int_{\mathbb{R}}\left(\left(k^{1}+a\right)^{2}+\lambda^{2}\right)^{-\frac{1}{2}}\left(k^{1^{2}}+\lambda^{2}\right)^{-\frac{1}{2}} d k^{1} \frac{\lambda d \lambda}{\left(1+\lambda^{2}\right)^{3 / 2}}
$$

Clearly $\left(\left(k^{1}+a\right)^{2}+\lambda^{2}\right)^{-\frac{1}{2}}$ and $\left(k^{1^{2}}+\lambda^{2}\right)^{-\frac{1}{2}} \in L^{2}\left(\mathbb{R}, d k^{1}\right)$ for $\lambda>0$. By the Cauchy Schwarz inequality we can dominate the inner integral by $\int_{\mathbb{R}}\left(k^{1^{2}}+\right.$ $\left.\lambda^{2}\right)^{-1} d k^{1}=\pi \lambda^{-1}$. Therefore, the above expression is smaller than

$$
2 \pi^{2} \int_{\mathbb{R}} \frac{d \lambda}{\left(1+\lambda^{2}\right)^{3 / 2}}<\infty
$$


independently of $\vec{a} \in \mathbb{R}^{3}$.

The estimate $\left|M_{n}^{n}(\varphi)\right| \leq C_{n}^{n}\|\varphi\|_{0,3}$ for a sufficiently large $C_{n}^{n}>0$ can be proved analogously.

Let us therefore consider the case $j=1, \cdots, n-1$. From the representation (20) we get the estimate

$$
\begin{aligned}
\left|M_{j}^{n}(\varphi)\right| \leq(2 \pi)^{3-n} 2^{-n} \int_{\mathbb{R}^{3 n-3}} \frac{\left|\sum_{l=1, l \neq j}^{n} \vec{k}_{l}\right|^{-1}\left|\vec{k}_{j+1}\right|^{-1}}{\left(1+\left|\vec{k}_{j+1}\right|^{2}\right)^{3 / 2}} \\
\times \prod_{l=1, l \neq j}^{n} \frac{\left|\vec{k}_{l}\right|^{-1}}{\left(1+\left|\vec{k}_{l}\right|^{2}\right)^{3 / 2}} \bigotimes_{l=1, l \neq j}^{n} d \vec{k}_{l}\|\varphi\|_{0,3}
\end{aligned}
$$

for $\varphi \in \mathcal{S}\left(\mathbb{R}^{d n}, \mathbb{C}\right)$. The integral can be dominated by the expression

$$
\left(\int_{\mathbb{R}^{3}} \frac{|\vec{k}|^{-1}}{\left(1+|\vec{k}|^{2}\right)^{3 / 2}} d \vec{k}\right)^{n-2}\left(\sup _{\vec{a} \in \mathbb{R}^{3}} \int_{\mathbb{R}^{3}} \frac{|\vec{k}+\vec{a}|^{-1}|\vec{k}|^{-1}}{\left(1+|\vec{k}|^{2}\right)^{3 / 2}} d \vec{k}\right),
$$

which is finite by the above calculations.

This completes the proof of the truncated Hilbert space structure condition on the truncated Wightman functions of the vector models.

Acknowledgements Stimulating discussions of the first named author with R. Gielerak, K. Iwata and T. Kolsrud are gratefully acknowledged. The financial support of D.F.G. (SFB 237) is also gratefully acknowledged. We also would like to thank the referee for very helpful comments and corrections on a previous version of this paper.

\section{References}

[1] Albeverio, S., Gottschalk, H., Wu, J.-L.: Euclidean random fields, pseudodifferential operators, and Wightman functions. pp. 20-37, in Stochastic Analysis and Applications(edts. Davies,I.M., Truman,A., Elworthy,K.D.). Singapore: World Scientific 1996.

[2] Albeverio, S., Gottschalk, H., Wu, J.-L.: Convoluted generalized white noise, Schwinger functions and their analytic continuation to Wightman functions. Bochum: SFB 237-Preprint Nr. 298, 1996, to appear in Rev. Math. Phys.

[3] Albeverio, S., Iwata, K., Kolsrud, T.: Random fields as solutions of the inhomogeneous quaternionic Cauchy-Riemann equation.I.Invariance and analytic continuation. Commun. Math. Phys. 132, 555-580 (1990).

[4] Albeverio, S., Wu, J.-L.: Euclidean random fields obtained by convolution from generalized white noise. J. Math. Phys. 36, 5217-5245 (1995). 
[5] Becker, C.: Reflection positivity for quantum vector fields. pp. 76-90, in Stochastic Analysis and Applications(edts. Davies,I.M., Truman,A., Elworthy,K.D.). Singapore: World Scientific 1996.

[6] Bognár, J.: Indefinite Inner Product Spaces. Berlin/Heidelberg/New York: Springer-Verlag 1974.

[7] Bogoliubov, N. N., Logunov, A. A., Todorov, R. T.: Introduction to Axiomatic Quantum Field Theory. Reading: Benjamin 1975(Translation and revision of original publication in 1969).

[8] Gelfand, I.M., Vilenkin, N. Ya.: Generalized Functions. II and IV. New York/London: Academic Press 1964.

[9] Gottschalk, H.: Die Momente gefalteten Gauss-Poissonschen Weißen Rauschens als Schwingerfunktionen. Diplomarbeit, Bochum 1995.

[10] Hida, T., Kuo, H.-H., Potthoff, J. and Streit, L.: White Noise: An Infinite Dimensional Calculus. Dordrecht/Boston/London: Kluwer Academic 1993.

[11] Jakobczyk, L., Strocchi, F.: Euclidean formulation of quantum field theory without positivity. Commun. Math. Phys. 119, 529-541 (1988).

[12] Jost, R.: The General Theory of Quantized Fields. Providence: AMS 1965.

[13] Morchio, G., Strocchi, F.: Infrared singularities, vacuum structure and pure phases in local quantum field theory. Ann. Inst. H. Poincaré A33, 251-282 (1980).

[14] Nelson, E.: Construction of quantum fields from Markoff fields. J. Funct. Anal. 12, 97-112 (1973).

[15] Osterwalder, K., Schrader, R.: Axioms for Euclidean Green's functions. I. Commum. Math. Phys. 31, 83-112 (1973); II. Commum. Math. Phys. 42, 281-305 (1975).

[16] Reed, M., Simon, B.: Methods of Modern Mathematical Physics. I. Functional Analysis. New York: Academic Press 1972; II. Fourier Analysis, Self-Adjointness. New York: Academic Press 1975; III. Scattering Theory. New York: Academic Press 1978.

[17] Simon, B.: The $P(\phi)_{2}$ Euclidean (Quantum) Field Theory. Princeton: Priceton University Press 1975.

[18] Streater, R. F., Wightman, A. S.: PCT, Spin and Statistics, and All That. New York: Benjamin 1964.

[19] Strocchi, F.: Selected Topics on the General Properties of Quantum Field Theory. Lect. Notes in Physics 51. Singapore/New York/ London/Hong Kong: World Scientific 1993. 